\title{
Finite Blocklength Non-Orthogonal Cooperative Communication Relying on SWIPT-enabled Energy Harvesting Relays
}

\author{
Akash Agarwal, Student Member, IEEE, Aditya K. Jagannatham, Member, IEEE, and Lajos Hanzo, Fellow, IEEE
}

\begin{abstract}
A finite blocklength (FBL) twin-user non-orthogonal cooperative downlink system is considered, wherein a base station simultaneously communicates with the users whilst relying on simultaneous wireless information and power transfer (SWIPT) enabled energy harvesting relay. Closed-form analytical expressions are obtained for the end-to-end average block error rate (BLER) assuming potentially dissimilar Nakagami- $m$ fading links. Furthermore, asymptotic expressions are derived for the individual user's end-to-end average BLER performance and diversity order at high signal to noise ratios (SNRs). Additionally, since, the stronger user's signal is detected, while assuming the weaker user's signal to be interference, an analytical bound has been obtained for characterizing the effect of this interference on the BLER performance of both users. Furthermore, a framework is developed for joint relay splitting factor and user power allocation towards end-to-end blocklength minimization under strict reliability constraints by converting the original non-convex optimization problem to a convex geometric program (GP) that can be solved efficiently. Simulation results are presented to validate the analytical expressions derived and illustrate the efficacy of the proposed scheme.
\end{abstract}

Index Terms-Non-orthogonal multiple access (NOMA), finite blocklength (FBL) communication, dissimilar Nakagami- $m$ fading links, energy harvesting, simultaneous wireless information and power transfer (SWIPT), product of Gamma random variables, geometric program (GP).

\section{INTRODUCTION}

Next-generation wireless communication networks are predicted to witness a significant increase in traffic all over the world [1]. Proliferation of internet of things (IoT) devices such as traffic sensors, autonomous machines, navigation and control systems are expected to contribute significantly to this increase. To support real-time mission critical applications in such environments, massive device connectivity [2] combined with low communication latency [3] and ultra-reliability [1] are expected to be the key design and performance features.

In this context, non-orthogonal techniques have gained significant interest as concept of supporting massive device

Akash Agarwal and Aditya K. Jagannatham are with the Department of Electrical Engineering, Indian Institute of Technology Kanpur, Kanpur 208016, U.P., India e-mail: (akash@iitk.ac.in; adityaj@iitk.ac.in).

Lajos Hanzo is with the School of Electronics and Computer Science, University of Southampton, Southampton SO17 1BJ, U.K. (E-mail: lh@ecs.soton.ac.uk).

This research has been supported in part by the IIMA IDEA Telecom Centre of Excellence (IITCOE) and Qualcomm Innovation Fellowship. L. Hanzo would like to acknowledge the financial support of the Engineering and Physical Sciences Research Council projects EP/Noo4558/1, EP/PO34284/1, COALESCE, of the Royal Society's Global Challenges Research Fund Grant as well as of the European Research Council's Advanced Fellow Grant QuantCom. connectivity [4], [5] at a low latency. Several users are multiplexed in the power domain over the same frequency and time resources improving the spectral efficiency. Subsequently, successive interference cancellation (SIC) is employed at the receiver to decode the individual signals of the multiplexed users [6], [7]. Hence, a large number of users/devices can be supported at any particular instant of time. A brief review of the recent research in non-orthogonal schemes and the motivation of the proposed work is presented next.

\section{A. Review of the Existing Literature}

Non-orthogonal downlink (DL) transmission increases the spectral efficiency [8], [9] not only as a standalone communication technique, but also in conjunction with several other existing communication techniques [7], [10], [11]. In particular, the outage probability of NOMA within a multipleinput multiple-output (MIMO) DL communication system has been explored by the authors of [12], [13]. User-power and subcarrier allocation has been studied for DL orthogonal frequency division multiplexing (OFDM) in [14]. The analysis of NOMA systems with cooperative one-way decode-andforward (DF) relaying has been reported in [9], [15], [16], whereas amplify-and-forward (AF) relaying for NOMA has been analyzed by Xue et. al. in [17]. Finally, the works in [18], [19], have extended the analysis of NOMA systems with two-way relays in the network. The above contributions have comprehensively demonstrated the improved spectral efficiency of NOMA in comparison to conventional orthogonal multiple access (OMA)-based techniques.

A limitation of the majority of NOMA studies is that they rely on classical Shannon capacity [20] analysis, which assumes having an arbitrarily low probability of error for very long transmit blocklengths. However, such large blocklengths are often not suitable for the IoT-NOMA networks of the future, since a large number of connected sensor devices often have to communicate using short bursts of data and the error probability is non-negligible due to the impact of the finite blocklength (FBL). Moreover, in FBL communication, the average BLER is strictly higher than the outage probability obtained using Shannon's approach [21]. Thus, the outage probability over-estimates the communication reliability at FBLs. This necessitates a fundamental shift from the conventional rate analysis to one that is more suited for the investigation of FBL communication systems.

Polyanskiy et al. [22] have derived the achievable rate and block error rate (BLER) expressions for FBL transmis- 
sions, which represents a paradigm shift from the Shannon capacity framework [20]. Facilitated by these results, the recent contributions [23]-[28] have explored the performance of NOMA employing a FBL communication framework, for various DL scenarios. In particular, the BLER analysis of a non-cooperative two-user DL communication scenario associated with Rayleigh fading channel links has been described in [24], [27]. Similar analysis for a multiple-user scenario associated with Nakagami- $m$ distributed fading channel links has been reported in [28]. Transmission rate optimization and energy minimization associated with reliability constraints at the receivers for two user DL communication systems has been reported in [25] and [23], respectively. The packet loss probability analysis of a similar non-cooperative DL system has been reported in [26].

Cooperative relaying has been shown to significantly improve both the system's reliability as well as the coverage area of wireless networks, especially for scenarios in which a direct path between the source and destination is not available. In this context, the performance analysis of OMA-based single-relay aided FBL communication scenarios has been presented in [29]-[32]. More recently, the performance analysis of OMAbased multiple-relay aided FBL communication systems has been reported in [33]. Finally, the contribution by Lai et. al. in [34] presents results for the BLER performance of a NOMA two-user DL system with user cooperation.

An additional challenge that can potentially limit the widespread deployment of IoT and other communication devices in next-generation networks is the supply of power [35], [36] to the connected devices, since the availability of conventional external power sources is limited. Hence, to address this problem, cooperative relaying employing energy harvesting [37] as well as simultaneous wireless information and power transfer (SWIPT) [38] can effectively address these challenges and ensure prolonged energy-sustainability in relay-aided communication networks [39], [40]. Since radio frequency (RF) signals can simultaneously carry both energy and information [41], SWIPT enabled relay/receivers can glean energy from the received signal for their future signal transmissions, while simultaneously decoding the received information symbols [42]. In addition, they have been shown to significantly improve the overall transmission performance [39], [43]-[45]. for large blocklength scenarios.

The average BLER analyses for point-to-point and DF relay-aided FBL communication systems have been reported in [46], [47] and [48], respectively, wherein energy transfer and information transmission take place in the downlink and uplink, respectively. In particular, the work in [48] considers also the circuit and signal processing power consumption at the relay. The work by Makki et. al. [49] has considered a SWIPT-based point-to-point system and analyzed the outage probability for FBL transmissions to an energy harvesting receiver. The energy supply probability and the achievable rate of point-to-point FBL transmissions relying on an energy harvesting transmitter has been studied by Khan et. al. in [50]. In the context of SWIPT-enabled energy harvesting OMAbased single-user relay-aided FBL communication, López et al. [51] analyze the error probability considering time splitting
SWIPT-based decode-and-forward (DF) relaying. By contrast, both time and power splitting-based SWIPT protocols have been studied by Haghifam et al. [52] for a similar system using amplify-and-forward (AF) relaying. In the recent work in [53], Hu et al. have presented an optimization framework for SWIPT parameter allocation with the goal of reliability maximization in a SWIPT-enabled DF relay-aided OMA-based single-user dual-hop FBL communication system.

The performance analysis reported in all of the abovementioned treatises on FBL communications have either considered a conventionally powered relay-aided OMA-based single user DL communication system [29]-[33] or an energy harvesting-enabled relay-aided single user OMA-based dualhop DL communication scenario [51]-[53]. Alternatively, simple point-to-point communication scenarios were considered, where either an energy harvesting-enabled source communicates with a single destination over FBL [46], [47], [50] or a conventionally powered source employs wireless energy transfer (WET) to enable information decoding at the receiver before information transmission [49]. Moreover, the contribution [48] described above and [51], [52] on SWIPT-enabled relay-aided single user DL OMA-based dual-hop communication have restricted their analyses to fixed blocklengths and/or power allocations, thus leaving the joint power allocation and blocklength optimization aspects unexplored. On the other hand, the authors of [53] solely focused their attention on the simplistic OMA-based single user communication scenario for instantaneous error minimization and resource allocation. Furthermore, the treatises relying on NOMA with FBL [23]-[28] have either considered conventionally powered non-cooperative two-user DL communication scenarios or a similarly powered two-user DL system with user cooperation [34]. Thus, to the best of our knowledge, none of the existing contributions analyze the performance of a non-orthogonal system relying on power splitting aided SWIPT-based energy harvesting relays using FBL. Moreover, since non-orthogonal communication techniques are spectrally efficient [7]-[11] while SWIPT is an energy-efficient solution [54], the analysis of non-orthogonal SWIPT-enabled FBL communication systems is of paramount importance in the context of nextgeneration systems. The salient contributions of this paper are described next.

\section{B. Our Contributions}

We consider a cooperative non-orthogonal DL communication system, where a base station (BS) transmits FBL data bursts to two users. Furthermore, similar to [51], [53], we assume the absence of a direct path due to intense shadowing or blockage between the BS and the users. Therefore, a powersplitting energy-harvesting DF relay is employed to support the process of transmission to the users. The system is spectrally efficient in comparison to conventional orthogonal transmission, since only a single phase is required for transmission of both the user's data to the relay. The choice of the DF protocol for relaying in this system can be justified as follows. DF relaying is capable of completely cleaning up the received signal before it's retransmission from a reduced distance with 
TABLE I: Comparative Literature Survey on NOMA, Relaying, SWIPT with FBL and the present work

\begin{tabular}{|c|c|c|c|c|c|c|c|c|c|c|c|c|c|}
\hline & [24], [27] & {$[28]$} & {$[23],[25],[26]$} & {$[29]-[33]$} & [34] & [50] & {$[46],[47]$} & [48] & [49] & [51] & [52] & [53] & Our Work \\
\hline NOMA-based system & $\checkmark$ & $\bar{v}$ & $\bar{v}$ & & $\bar{s}$ & & & & & & & & $\checkmark$ \\
\hline Cooperative communication & & & & $\checkmark$ & $\checkmark$ & & & $\checkmark$ & & $\checkmark$ & $\checkmark$ & $\checkmark$ & $\checkmark$ \\
\hline DF Relaying & & & & $\checkmark$ & $\checkmark$ & & & $\checkmark$ & & $\checkmark$ & & $\checkmark$ & $\checkmark$ \\
\hline AF Relaying & & & & & & & & & & & $\checkmark$ & & \\
\hline Energy Harvesting at information Source/Destination & & & & & & $\checkmark$ & $\checkmark$ & $\checkmark$ & $\checkmark$ & & & & \\
\hline Energy Harvesting at Relay & & & & & & & & $\checkmark$ & & $\checkmark$ & $\checkmark$ & $\checkmark$ & $\checkmark$ \\
\hline Power Splitting SWIPT at Relay & & & & & & & & & & & $\checkmark$ & $\checkmark$ & $\checkmark$ \\
\hline Average BLER analysis & $\checkmark$ & $\checkmark$ & & & $\checkmark$ & & $\checkmark$ & $\checkmark$ & & $\checkmark$ & $\checkmark$ & & $\checkmark$ \\
\hline User Optimum/Near-optimum power allocation & $\checkmark$ & & $\checkmark$ & & & $\checkmark$ & & & $\checkmark$ & & & & $\checkmark$ \\
\hline Blocklength Minimization & $\checkmark$ & & & & & & & & & & & & $\checkmark$ \\
\hline $\begin{array}{l}\text { Achievable Rate/Packet loss/Instant. Error/Reliability/ } \\
\text { Outage/Throughput/Energy Use analysis and/or optimization }\end{array}$ & & & $\checkmark$ & $\checkmark$ & & $\checkmark$ & & $\checkmark$ & $\checkmark$ & $\checkmark$ & & $\checkmark$ & \\
\hline Diversity Order Analysis & & & & & & & & & & & & & $\checkmark$ \\
\hline Impact of Superposition on BLER & & & & & & & & & & & & & $\checkmark$ \\
\hline
\end{tabular}

respect to the destination, provided that the SNR is sufficiently high. By contrast, AF relaying is unable to improve the SNR owing to noise amplification [55]-[57]. Moreover, AF relaying will necessitate CSI acquisition of the source-relay link at $D_{1}, D_{2}$, thus leading to higher communication overheads in comparison to the DF technique that does not require this. The pivotal contributions of this paper are itemized below:

- A power splitting SWIPT-based energy harvesting DF relay-aided non-orthogonal cooperative communication scheme has been considered wherein a source transmits data to the two-users with transmissions over FBL and possibly dissimilar Nakagami- $m$ fading links.

- Expressions have been obtained for the average BLER using a novel approximation for the CDF of the product of two independent Gamma random variables. Furthermore, results have also been presented for the diversity orders of the users.

- An asymptotic bound has been derived to characterize the effect of the interference due to superposition of user symbols on the end-to-end average BLER at both the users for FBLs.

- A novel geometric programming based convex optimization framework is developed for joint total BS and relay blocklength minimization together with evaluation of the source and relay power allocation and the SWIPTsplitting factor at the relay, subject to user reliability constraints.

- Finally, simulation results are presented to illustrate the benefits of the proposed scheme over conventional OMA with SWIPT and also non-orthogonal WET-based scheme wherein energy transfer and information transmission take place in different phases. The results also lend credence to the various analytical expressions derived in the paper.

The rest of the paper is organized as follows. The system model is described in Section II, while the instantaneous and average end-to-end BLER expressions of the two users have been obtained in Section III-A and III-B, respectively. This is followed by the expressions of the asymptotic average BLERs and the diversity orders at high SNRs as well as by the characterization of the BLER contribution arising due to the interference imposed by the weaker signal in Section IV. Our GP based optimization framework developed for the total joint end-to-end blocklength, for the source/relay power and splitting factors under strict user-reliability constraints in
Section V. Our simulation results are presented in Section VI, followed by our conclusions in Section VII.

The notation used in this paper is summarized next. The quantity $\mathcal{G}(a, b)$ represents a Gamma distributed random variable with $a$ and $b$ as shape and rate parameters, respectively. $\mathcal{C N}\left(0, \sigma^{2}\right)$ represents a zero-mean symmetric complex Gaussian with variance $\sigma^{2} . f_{X}(\cdot)$ and $F_{X}(\cdot)$ denote the probability distribution function (PDF) and the cumulative distribution function $(\mathrm{CDF})$ of random variable $X$, respectively. $\operatorname{Pr}(\cdot)$ denotes the probability of an event. $\Gamma(x+1)=x$ ! denotes the Gamma function and $\Gamma(s, x)=\int_{x}^{\infty} x^{s-1} e^{-x} d x, \bar{\Gamma}(s, x)=$ $\int_{0}^{x} x^{s-1} e^{-x} d x$ denote the standard upper, lower incomplete Gamma function, respectively. $\mathcal{R}^{+}$denotes the set of positive real numbers, $\mathbb{E}(\cdot)$ denotes the expectation operator, $\min (\cdot, \cdot)$ denotes the minimum operator and underlined boldface symbols such as $\underline{\xi}$ denote vectors.

\section{SySTEM MODEL}

Consider the non-orthogonal DL of Fig. 1 where the BS $S$ transmits to the destinations $D_{1}$ and $D_{2}$ assisted by a single conventional DF relay $R$, which decodes the received symbol, re-encodes and forwards to the destination. Similar to [51] [53], the direct path between the base station and the users is assumed to be absent due to intense shadowing or blockage. Furthermore, similar to [24], [28], [51], [52], all the terminals are considered to be half-duplex with a single antenna each. Such a system is well suited for IoT scenarios, where the required data rates are low and single antenna systems are preferred due to their low complexity. The source $S$ is assumed to be powered by an external source, whereas the relay $R$ employs power splitting SWIPT-based energy harvesting prior to transmission to the two users. Only the statistical CSI of all the links is assumed to be available at the $B S$, whereas the relay has access to only the CSI statistics of the relay destination links, but not to the actual fading gains of $D_{i}$. The users are ordered as $\lambda_{R D_{1}} \leq \lambda_{R D_{2}}$, where $\lambda_{R D_{1}}$ and $\lambda_{R D_{2}}$ are the average channel gains of the relay-destination links i.e. $\lambda_{R D_{i}}=\mathbb{E}\left(\left|h_{R D_{i}}\right|^{2}\right), i \in\{1,2\}$. Hence, statistically, $D_{2}$ is the user having a better channel, while user $D_{1}$ has a weaker channel. Accordingly, $D_{1}$ will be allocated a higher power and it's signal will always be decoded first [58], [59]. Communication between $S$ and $D_{1}, D_{2}$ takes places over two phases as described below. 


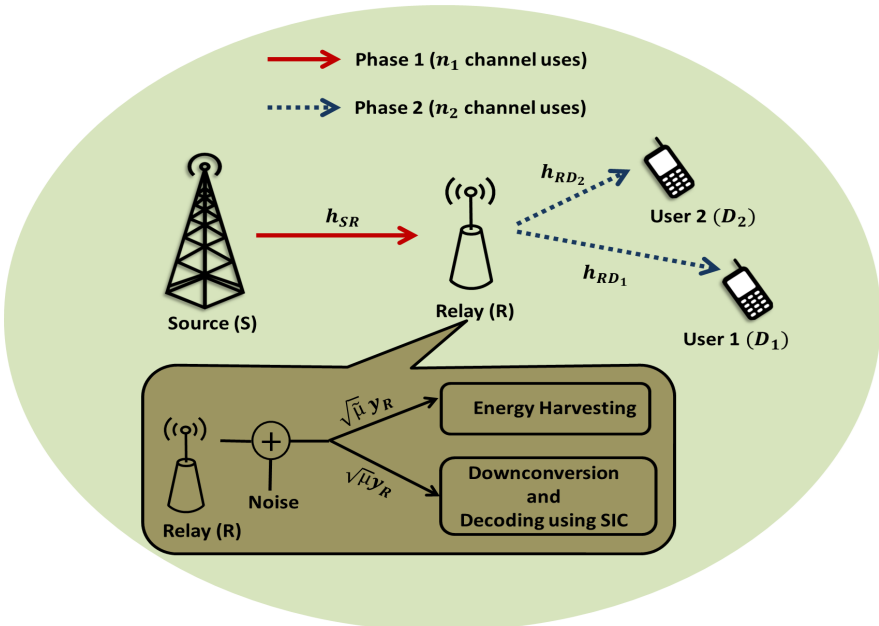

Fig. 1: System Model.

\section{A. Phase 1}

In Phase 1, the BS $S$ employs SWIPT for the transfer of both energy and information symbols to $R$ over $n_{1}$ channel uses. In particular, $S$ linearly combines the symbols $x_{1}, x_{2}$ destined for $D_{1}, D_{2}$, respectively, followed by transmission of the resultant superposed signal to $R$. The RF signal received at $R$ can be mathematically represented as

$$
y_{R}=h_{S R}\left(\sqrt{a_{1} P_{S}} x_{1}+\sqrt{a_{2} P_{S}} x_{2}\right)+w_{R}
$$

where, as described in [58], [59] for a non-orthogonal downlink scenario, we have $a_{1} \geq a_{2}>0$, so that $a_{1}+a_{2}=1$, are the power factors that denote the fractions of the total power allocated to $D_{1}, D_{2}$, respectively. Thus, the user having a weaker channel is allocated a higher fraction of the total power. The quantity $h_{S R}$ is the Nakagami- $m$ distributed channel coefficient between $S$ and $R$, which represents the combined effects of small scale fading as well as path loss, with the fading parameter $m_{S R}$ assumed to be a positive integer [59], [60] and $\mathbb{E}\left|h_{S R}\right|^{2}=\lambda_{S R}$ being the average channel gain of the $S-R$ link. Consequently, the channel gain is Gamma distributed as $\left|h_{S R}\right|^{2} \sim \mathcal{G}\left(\alpha_{s r}, \beta_{s r}\right)$, where $\alpha_{S R}=m_{S R}$ and $\beta_{S R}=\frac{\alpha_{S R}}{\lambda_{S R}}>0$ denote the shape and rate parameters ${ }^{1}$, respectively. This model is general, hence it can also be used for the analysis of Rayleigh fading channels as a special case by setting $m_{S R}=1$. The quantity $w_{R}$ denotes the additive white Gaussian noise at $R$ that is distributed as $w_{R} \sim \mathcal{C N}\left(0, \sigma^{2}\right)$.

The relay employs power splitting similar to [61] for partitioning the received signal $y_{R}$ into two components, one for information transmission and one for energy transfer and denoted by $y_{R}^{I}=\sqrt{\mu} y_{R}, y_{R}^{E}=\sqrt{\tilde{\mu}} y_{R}$, where the quantities $\mu, \tilde{\mu}>0, \mu+\tilde{\mu} \leq 1$, denote the information decoding and power splitting coefficients, respectively. Thus, similar to other existing contributions [51]-[53], the energy harvested at $R$ can be expressed as

$$
E_{R}=\eta \tilde{\mu} P_{S}\left|h_{S R}\right|^{2} n_{1}
$$

\footnotetext{
${ }^{1}$ Shape parameter is a measure of the statistical dispersion of the distribution curve. Rate parameter is the reciprocal of the scale parameter which characterizes the spread of the distribution.
}

where $0<\eta<1$ denotes the energy conversion efficiency [40]. As seen from (2), in addition to the channel gain, the amount of harvested energy depends also on the tunable parameters $\tilde{\mu}$ and $n_{1}$, which can be appropriately chosen to maximize the energy harvested. This is further explored in the optimization problem of Section V. Furthermore, a better approximation for the energy harvesting model is given by a piece-wise linear function [62] expressed as

$$
E_{R}= \begin{cases}0, & \tilde{\mu} P_{S}\left|h_{S R}\right|^{2}<\Xi_{\min } \\ \eta \tilde{\mu} P_{S}\left|h_{S R}\right|^{2} n_{1}, & \Xi_{\min } \leq \tilde{\mu} P_{S}\left|h_{S R}\right|^{2} \leq \Xi_{\max }, \\ \eta \Xi_{\max } n_{1}, & \text { otherwise, }\end{cases}
$$

where $\Xi_{\min }, \Xi_{\max }$ are the activation or sensitivity and saturation thresholds, respectively. The BLER analysis corresponding to this alternative model, including also the power consumed during the SIC process is presented in Section $\mathrm{X}$ of our technical report in [63]. Subsequently, in order to decode the information bits from the received RF signal, the relay $R$ initially downconverts $y_{R}^{I}$ to obtain the baseband information signal

$$
\tilde{y}_{R}^{I}=h_{S R}\left(\sqrt{a_{1} \mu P_{S}} x_{1}+\sqrt{a_{2} \mu P_{S}} x_{2}\right)+\sqrt{\mu} w_{R}+\tilde{w}_{R},
$$

where $\tilde{w}_{R} \sim \mathcal{C N}\left(0, \tilde{\sigma}^{2}\right)$, denotes the additive noise at the output of the downconverter [39]. Thereafter, SIC is employed to decode the symbols $x_{1}$ and $x_{2}$ corresponding to users $D_{1}$ and $D_{2}$, respectively. In particular, $R$ first decodes the statistically stronger signal of user $D_{1}$ considering the other signal components as noise. Let $\gamma_{i}^{U}$ denote the signal to interference plus noise ratio (SINR) for decoding $x_{i}$ at terminal $U, i \in\{1,2\}, U \in\left\{R, D_{1}, D_{2}\right\}$. Thus, the SINR of decoding $x_{1}$ at $R$ can be written as

$$
\gamma_{1}^{R}=\frac{a_{1} \mu P_{S}\left|h_{s r}\right|^{2}}{a_{2} \mu P_{S}\left|h_{S R}\right|^{2}+\mu \sigma^{2}+\tilde{\sigma}^{2}} \approx \frac{a_{1} \mu \rho_{S}\left|h_{s r}\right|^{2}}{a_{2} \mu \rho_{S}\left|h_{S R}\right|^{2}+1},
$$

where $\rho_{S}=\frac{P_{S}}{\tilde{\sigma}^{2}}$ and $\sigma^{2}$ has been neglected since in practice $\sigma^{2} \ll \tilde{\sigma}^{2}$ [39]. After decoding the stronger signal component, the relay remodulates it and subtracts it from the composite signal, leaving behind the statistically weaker signal of $D_{2}$ [7]. Assuming that $R$ decodes $x_{1}$ correctly, the signal to noise ratio (SNR) of decoding $x_{2}$ at $R$ can be written as

$$
\gamma_{2}^{R}=a_{2} \mu \rho_{S}\left|h_{S R}\right|^{2} .
$$

\section{B. Phase 2}

In this phase, the relay $R$ superposes the decoded symbols $\hat{x}_{1}, \hat{x}_{2}$, which can potentially be in error, and broadcasts the signal obtained to both $D_{1}$ and $D_{2}$. In the event that the relay has decoded the received symbols $x_{1}, x_{2}$ correctly in the first phase, the signal received by the two users can be written as

$y_{D_{i}}=h_{R D_{i}}\left(\sqrt{b_{1} P_{R}} x_{1}+\sqrt{b_{2} P_{R}} x_{2}\right)+w_{D_{i}}, i \in\{1,2\}$,

where, $b_{1} \geq b_{2}>0$, with $b_{1}+b_{2}=1$, are the relay power factors corresponding to $D_{1}, D_{2}$, respectively. Thus, similar to source-relay transmission, the user having a weaker channel is once again allotted a larger fraction of the total power. The term $h_{R D_{i}}$ denotes the Nakagami- $m$ fading channel coefficient 
between the relay and each user $D_{i}$, representing the combined effects of small scale fading, as well as path loss [59], with the fading parameters denoted by $m_{R D_{i}}$ being positive integers and $\mathbb{E}\left(\left|h_{R D_{i}}\right|^{2}\right)=\lambda_{R D_{i}}>0$ being the average channel gains for the relay-destination links. Thus, the channel gains are Gamma distributed $\left|h_{R D_{i}}\right|^{2} \sim \mathcal{G}\left(\alpha_{R D_{i}}, \beta_{R D_{i}}\right)$ with $\alpha_{R D_{i}}=$ $m_{R D_{i}}$ and $\beta_{R D_{i}}=\frac{\alpha_{R D_{i}}}{\lambda_{R D_{i}}}>0$ denoting the corresponding shape and rate parameters, respectively. Furthermore, based on the assumption that $D_{2}$ has the stronger channel, we have $\lambda_{R D_{2}} \geq \lambda_{R D_{1}}$. Finally, $P_{R}$ denotes the relay's transmit power obtained from (2) as

$$
P_{R}=\frac{E_{R}}{n_{2}}=\frac{\eta \tilde{\mu} n_{1} P_{S}\left|h_{S R}\right|^{2}}{n_{2}},
$$

where, in order to simplify the analysis to the most salient aspects of energy harvesting systems, the power consumed during information and signal processing has been neglected, which is an idealized simplifying assumption that has been adopted in the authoritative treatises such as [39], [51], [53]. Moreover, the BLER analysis including also the power consumed during SIC is presented in Section $\mathrm{X}$ of our technical report in [63] due to lack of space. Accordingly, after receiving the signal, $D_{1}$ decodes $x_{1}$ directly, treating the rest of the signal components as noise, and after subtracting the remodulated signal, $D_{2}$ automatically acquires it's own symbol $x_{2}$. Thus, the SINRs of decoding $x_{1}$ at the two users can be expressed as

$$
\begin{aligned}
\gamma_{1}^{D_{i}} & =\frac{b_{1} P_{R}\left|h_{R D_{i}}\right|^{2}}{b_{2} P_{R}\left|h_{R D_{i}}\right|^{2}+\sigma^{2}} \\
& =\frac{b_{1} \eta \tilde{\mu} n_{1} \rho_{S}\left|h_{S R}\right|^{2}\left|h_{R D_{i}}\right|^{2}}{b_{2} \eta \tilde{\mu} n_{1} \rho_{S}\left|h_{S R}\right|^{2}\left|h_{R D_{i}}\right|^{2}+n_{2}},
\end{aligned}
$$

where $i \in\{1,2\}$. Subsequently, after correctly decoding $x_{1}$ at $D_{2}, x_{2}$ can be decoded at an SNR

$$
\gamma_{2}^{D_{2}}=\frac{b_{2} P_{R}\left|h_{R D_{2}}\right|^{2}}{\sigma^{2}}=\frac{b_{2} \eta \tilde{\mu} n_{1} \rho_{S}\left|h_{S R}\right|^{2}\left|h_{R D_{2}}\right|^{2}}{n_{2}} \text {. }
$$

\section{InStANTANEOUS AND AVERAGE BLOCK ERROR ANALYSIS}

\section{A. Instantaneous BLERs at $D_{1}$ and $D_{2}$}

Let $N_{i}$ be the number of bits to be transmitted to users $D_{i}$. Let $\phi_{i}^{U}$ denote the event that the block corresponding to user $i, i \in\{1,2\}$, is decoded in error at terminal $U, U \in$ $\left\{R, D_{1}, D_{2}\right\}$. Furthermore, let $\bar{\phi}_{i}^{U}$ denote the complement of the event $\phi_{i}^{U}$. From the results of [22], the instantaneous BLER $\tilde{\epsilon}$ for a given SNR $\gamma$ and FBL of size $n, n \geq 100$, is given by

$$
\tilde{\epsilon} \approx Q\left(\frac{n \log _{2}(1+\gamma)-N}{\sqrt{n V(\gamma)}}\right),
$$

where $\log _{2}(1+\gamma)$ is the Shannon capacity, $N$ denotes the number of bits to be transmitted to the user, $V(\gamma)=$ $\left(1-\frac{1}{(1+\gamma)^{2}}\right)\left(\log _{2} e\right)^{2}$ represents the channel's dispersion coefficient and $Q$ is the Gaussian Q-function defined as $Q(x)=\frac{1}{\sqrt{2 \pi}} \int_{x}^{\infty} e^{\frac{-t^{2}}{2}} d t$. The above result is now exploited for evaluating the BLER for decoding $x_{i}$ at the various nodes in the system under consideration.
The instantaneous BLER for decoding $x_{1}$ at $R$, can be formulated as

$$
\operatorname{Pr}\left(\phi_{1}^{R}\right)=\tilde{\epsilon}_{1}^{R} \approx Q\left(\frac{n_{1} \log _{2}\left(1+\gamma_{1}^{R}\right)-N_{1}}{\sqrt{n_{1} V\left(\gamma_{1}^{R}\right)}}\right),
$$

where the SINR $\gamma_{1}^{R}$ is given in (5). Subsequently, assuming that $R$ decodes and then cancels the signal component corresponding to $x_{1}$ in (4) correctly, the BLER of decoding $x_{2}$ subsequently at $R$ is determined as

$$
\operatorname{Pr}\left(\phi_{2}^{R} \mid \bar{\phi}_{1}^{R}\right)=\tilde{\epsilon}_{2}^{R} \approx Q\left(\frac{n_{1} \log _{2}\left(1+\gamma_{2}^{R}\right)-N_{2}}{\sqrt{n_{1} V\left(\gamma_{2}^{R}\right)}}\right),
$$

where $\gamma_{2}^{R}$ has been defined in (6). Note that SIC can only be performed successfully at $R$ when $x_{1}$ is decoded correctly. Hence, due to the high level of interference, it follows that $x_{2}$ will be decoded in error when $x_{1}$ is decoded incorrectly. Therefore, $\operatorname{Pr}\left(\phi_{2}^{R} \mid \phi_{1}^{R}\right)$ is assumed to be equal to unity. Upon exploiting this fact, the net BLER of decoding $x_{2}$ at $R$ can be approximated as

$$
\begin{aligned}
& \operatorname{Pr}\left(\phi_{2}^{R}\right)=\operatorname{Pr}\left(\phi_{2}^{R} \mid \phi_{1}^{R}\right) \operatorname{Pr}\left(\phi_{1}^{R}\right)+\operatorname{Pr}\left(\phi_{2}^{R} \mid \bar{\phi}_{1}^{R}\right) \operatorname{Pr}\left(\bar{\phi}_{1}^{R}\right) \\
& \Longrightarrow \epsilon_{2}^{R} \approx 1 \times \tilde{\epsilon}_{1}^{R}+\tilde{\epsilon}_{2}^{R} \times\left(1-\tilde{\epsilon}_{1}^{R}\right)=\tilde{\epsilon}_{1}^{R}+\tilde{\epsilon}_{2}^{R}-\tilde{\epsilon}_{2}^{R} \tilde{\epsilon}_{1}^{R},
\end{aligned}
$$

where $\operatorname{Pr}\left(\phi_{2}^{R} \mid \phi_{1}^{R}\right)$ denotes the conditional probability of $\phi_{2}^{R}$ given $\phi_{1}^{R}$ and the event $\phi_{1}^{R}$ represents erroneous SIC at the relay. Furthermore, since in ultra-reliable communications the individual error terms $\tilde{\epsilon}_{2}^{R}, \tilde{\epsilon}_{1}^{R}$ are typically small and generally in the range of $10^{-3}$ to $10^{-5}$ [3], [24], the product term $\tilde{\epsilon}_{2}^{R} \tilde{\epsilon}_{1}^{R}$ above can be ignored without any significant impact on the analytical results. Thus, the error expression in (14) can be further approximated as

$$
\begin{aligned}
\epsilon_{2}^{R} \approx \tilde{\epsilon}_{1}^{R}+\tilde{\epsilon}_{2}^{R} \approx Q & \left(\frac{n_{1} \log _{2}\left(1+\gamma_{1}^{R}\right)-N_{1}}{\sqrt{n_{1} V\left(\gamma_{1}^{R}\right)}}\right) \\
& +Q\left(\frac{n_{1} \log _{2}\left(1+\gamma_{2}^{R}\right)-N_{2}}{\sqrt{n_{1} V\left(\gamma_{2}^{R}\right)}}\right) .
\end{aligned}
$$

In the next phase, the relay $R$ superposes the decoded symbols $x_{1}, x_{2}$ and broadcasts the signal obtained to both the users $D_{1}, D_{2}$, as described in Section II-B. After receiving this superposed signal, $D_{1}$ decodes the symbol $x_{1}$ directly, whereas $D_{2}$ employs SIC to obtain the desired symbol $x_{2}$. Proceeding in a manner similar to the one adopted for obtaining the BLER for decoding $x_{2}$ at $R$ in (15), one can obtain the net end-toend BLERs for decoding $x_{1}$ at $D_{1}$ and $x_{2}$ at $D_{2}$, denoted by $\epsilon^{D_{1}}, \epsilon^{D_{2}}$, in (16),(17), respectively, where we have assumed that the symbol $x_{i}$ cannot be decoded correctly at $D_{i}$, unless it has been decoded correctly at $R$ that is $\operatorname{Pr}\left(\phi_{i}^{D_{i}} \mid \phi_{i}^{R}\right)=1$. Thus, the BLER analysis accounts for the event of decoding error at the relay. Furthermore, it has also been assumed that $x_{2}$ cannot be decoded correctly at $D_{2}$, unless $x_{1}$ has been decoded correctly, which implies $\operatorname{Pr}\left(\phi_{2}^{D_{2}} \mid \phi_{1}^{D_{2}}\right)=1$. 


$$
\begin{aligned}
\epsilon^{D_{1}} & =\operatorname{Pr}\left(\phi_{1}^{D_{1}} \mid \phi_{1}^{R}\right) \times \operatorname{Pr}\left(\phi_{1}^{R}\right)+\operatorname{Pr}\left(\phi_{1}^{D_{1}} \mid \bar{\phi}_{1}^{R}\right) \times \operatorname{Pr}\left(\bar{\phi}_{1}^{R}\right)=1 \times \operatorname{Pr}\left(\phi_{1}^{R}\right)+\operatorname{Pr}\left(\phi_{1}^{D_{1}} \mid \bar{\phi}_{1}^{R}\right) \times\left(1-\operatorname{Pr}\left(\phi_{1}^{R}\right)\right) \\
& \approx \operatorname{Pr}\left(\phi_{1}^{R}\right)+\operatorname{Pr}\left(\phi_{1}^{D_{1}} \mid \bar{\phi}_{1}^{R}\right)=\tilde{\epsilon}_{1}^{R}+\tilde{\epsilon}_{1}^{D_{1}} \approx Q\left(\frac{n_{1} \log _{2}\left(1+\gamma_{1}^{R}\right)-N_{1}}{\sqrt{n_{1} V\left(\gamma_{1}^{R}\right)}}\right)+Q\left(\frac{n_{2} \log _{2}\left(1+\gamma_{1}^{D_{1}}\right)-N_{1}}{\sqrt{n_{2} V\left(\gamma_{1}^{D_{1}}\right)}}\right), \\
\epsilon^{D_{2}} & \approx \epsilon_{2}^{R}+\operatorname{Pr}\left(\phi_{1}^{D_{2}}\right)+\operatorname{Pr}\left(\phi_{2}^{D_{2}} \mid \bar{\phi}_{1}^{D_{2}}\right) \operatorname{Pr}\left(\bar{\phi}_{1}^{D_{2}}\right) \approx \epsilon_{2}^{R}+\tilde{\epsilon}_{1}^{D_{2}}+\operatorname{Pr}\left(\phi_{2}^{D_{2}} \mid \bar{\phi}_{1}^{D_{2}}\right)=\epsilon_{2}^{R}+\tilde{\epsilon}_{1}^{D_{2}}+\tilde{\epsilon}_{2}^{D_{2}} \\
& \approx \sum_{j=1}^{2} Q\left(\frac{n_{1} \log _{2}\left(1+\gamma_{j}^{R}\right)-N_{j}}{\sqrt{n_{1} V\left(\gamma_{j}^{R}\right)}}\right)+Q\left(\frac{n_{2} \log _{2}\left(1+\gamma_{j}^{D_{2}}\right)-N_{j}}{\sqrt{n_{2} V\left(\gamma_{j}^{D_{2}}\right)}}\right),
\end{aligned}
$$

\section{B. Average BLERs at $D_{1}$ and $D_{2}$}

Using (16),(17), the average BLER of decoding $x_{1}$ at $D_{1}$, $x_{2}$ at $D_{2}$ can be approximated as

$$
\begin{aligned}
& \mathbb{E}\left(\epsilon^{D_{1}}\right) \approx \mathbb{E}\left(\tilde{\epsilon}_{1}^{R}\right)+\mathbb{E}\left(\tilde{\epsilon}_{1}^{D_{1}}\right) . \\
& \mathbb{E}\left(\epsilon^{D_{2}}\right) \approx \mathbb{E}\left(\tilde{\epsilon}_{1}^{R}\right)+\mathbb{E}\left(\tilde{\epsilon}_{2}^{R}\right)+\mathbb{E}\left(\tilde{\epsilon}_{1}^{D_{2}}\right)+\mathbb{E}\left(\tilde{\epsilon}_{2}^{D_{2}}\right) .
\end{aligned}
$$

To determine $\mathbb{E}\left(\epsilon^{D_{1}}\right)$ and $\mathbb{E}\left(\epsilon^{D_{2}}\right)$, we first obtain $\mathbb{E}\left(\tilde{\epsilon}_{1}^{R}\right), \mathbb{E}\left(\tilde{\epsilon}_{1}^{D_{i}}\right)$, followed by $\mathbb{E}\left(\tilde{\epsilon}_{2}^{R}\right), \mathbb{E}\left(\tilde{\epsilon}_{2}^{D_{2}}\right)$, and subsequently substitute them in (18),(19) to obtain the average endto-end BLER at $D_{1}$ and $D_{2}$. However, the direct evaluation of the expected value of $\epsilon^{D_{i}}$ is mathematically intractable. Therefore, for mathematical tractability, the expression of the instantaneous BLER is approximated as [64]

$$
\tilde{\epsilon}_{i}^{U} \approx \begin{cases}1, & \gamma_{i}^{U} \leq \zeta_{i}^{U} \\ \frac{1}{2}-\tau_{i}^{U} \sqrt{n_{j}}\left(\gamma_{i}^{U}-\psi_{i}^{U}\right), & \zeta_{i}^{U}<\gamma_{i}^{U}<\Delta_{i}^{U} \\ 0, & \gamma_{i}^{U} \geq \Delta_{i}^{U},\end{cases}
$$

where we have

$$
\begin{aligned}
& \tau_{i}^{U}=\left(2 \pi\left(2^{\frac{2 N_{i}}{n_{j}}}-1\right)\right)^{\frac{-1}{2}}, \psi_{i}^{U}=2^{\frac{N_{i}}{n_{j}}}-1, \\
& \zeta_{i}^{U}=\psi_{i}^{U}-\frac{1}{2 \tau_{i}^{U} \sqrt{n_{j}}}, \Delta_{i}^{U}=\psi_{i}^{U}+\frac{1}{2 \tau_{i}^{U} \sqrt{n_{j}}},
\end{aligned}
$$

where $j=1$ when $U=R$ and $j=2$, otherwise. Evaluating the expectation of (20) to obtain the average BLER yields

$$
\mathbb{E}\left(\tilde{\epsilon}_{i}^{U}\right) \approx \tau_{i}^{U} \sqrt{n_{j}} \int_{\zeta_{i}^{U}}^{\Delta_{i}^{U}} F_{\gamma_{i}^{U}}(x) d x .
$$

The analytical results for the average BLER performance of both the users are presented next.

In order to determine $\mathbb{E}\left(\tilde{\epsilon}_{1}^{R}\right)$ using (22), the CDF of $\gamma_{1}^{R}$ can be evaluated as shown next. Starting from the expression in (5) for $\gamma_{1}^{R}$, it follows that

$$
\begin{aligned}
F_{\gamma_{1}^{R}}(x) & =\operatorname{Pr}\left(\gamma_{1}^{R} \leq x\right)=\operatorname{Pr}\left(\frac{a_{1} \mu \rho_{S}\left|h_{s r}\right|^{2}}{a_{2} \mu \rho_{S}\left|h_{S R}\right|^{2}+1} \leq x\right) \\
& =\frac{\bar{\Gamma}\left(\alpha_{S R}, \frac{\beta_{S R} x}{\mu \rho_{S}\left(a_{1}-x a_{2}\right)}\right)}{\Gamma\left(\alpha_{S R}\right)}, x<\frac{a_{1}}{a_{2}} .
\end{aligned}
$$

Substituting $F_{\gamma_{1}^{R}}(x)$ in (22), we can simplify the expression for $\mathbb{E}\left(\tilde{\epsilon}_{1}^{R}\right)$ as shown in Section I of our technical report in [63] to obtain the final expression of $\mathbb{E}\left(\tilde{\epsilon}_{1}^{R}\right)$ as given in (24). Furthermore, once again invoking (22), the average BLER of decoding $x_{1}$ at $D_{i}$ in the second phase, denoted by $\mathbb{E}\left(\tilde{\epsilon}_{1}^{D_{i}}\right)$ can be written as

$$
\mathbb{E}\left(\tilde{\epsilon}_{1}^{D_{i}}\right) \approx \tau_{1}^{D_{i}} \sqrt{n_{2}} \int_{\zeta_{1}^{D_{i}}}^{\Delta_{1}^{D_{i}}} F_{\gamma_{1}^{D_{i}}}(x) d x,
$$

where $\gamma_{1}^{D_{i}}=\frac{b_{1} \eta \tilde{\mu} n_{1} \rho_{S}\left|h_{S R}\right|^{2}\left|h_{R D_{i}}\right|^{2}}{b_{2} \eta \tilde{\mu} n_{1} \rho_{S}\left|h_{S R}\right|^{2}\left|h_{R D_{i}}\right|^{2}+n_{2}}$ as defined in (9). The next result yields a tractable approximation for the CDF of the product of two Gamma distributed random variables, which is employed in the subsequent analysis.

Theorem 1. Let $X_{i} \sim \mathcal{G}\left(\alpha_{i}, \beta_{i}\right), \quad i \in\{1,2\}$, be two independent Gamma distributed random variables such that $\alpha_{1}, \alpha_{2}, \beta_{1}, \beta_{2} \in \mathcal{R}^{+}$and $\alpha_{1}-\alpha_{2} \neq \pm \frac{1}{2}, \pm \frac{3}{2}, \cdots$. Then, the $C D F F_{Z}(z)$ of the random variable $Z$, where $Z=X_{1} X_{2}$, can be approximated as $F_{Z}(z) \approx$ $\sum_{v=0}^{\tilde{v}} \sum_{k=0}^{v} w_{v, k} \bar{\Gamma}\left(s_{k}, \sqrt{4 \beta_{1} \beta_{2} z}\right)$, where $\tilde{v}$ is a constant and can be chosen depending on the desired accuracy of the approximation, $s_{k}=2 \tilde{s}+k$,

$$
\tilde{s}= \begin{cases}\min \left(\alpha_{1}, \alpha_{2}\right), & \alpha_{1} \neq \alpha_{2} \\ \alpha-1, & \alpha_{1}=\alpha_{2}=\alpha,\end{cases}
$$

$w_{v, k}=\frac{\Lambda\left(\left|\alpha_{1}-\alpha_{2}\right|, v, k\right)}{\Gamma\left(\alpha_{1}\right) \Gamma\left(\alpha_{2}\right) 2^{\alpha_{1}+\alpha_{2}-2}}$. The constant $\Lambda\left(\left|\alpha_{1}-\alpha_{2}\right|, v, k\right)$ is defined in (27) where the quantities $L(v, k)$ denote the Lah numbers [65] that are defined as $L(0,0)=1, L(v, 0)=$ $0, L(v, 1)=v !$ and $\left(\begin{array}{c}v-1 \\ k-1\end{array}\right) \frac{v !}{k !}$ otherwise, for $v, k>0$.

Proof. A detailed proof is given in Section II of our technical report in [63].

Using the result in Theorem 1 to obtain $F_{\gamma_{1}^{D_{i}}}(x)$ followed by it's substitution into (25) and further simplification as shown in Section III of our technical report in [63] the average BLER for decoding $x_{1}$ at $D_{i}$ can be expressed as given in (28), where the term $\hat{I}_{i, l}^{D_{i}}$ is defined in Section III of our technical report in [63]. Note that the expression in (28) is tight even for small and moderate values of $\tilde{v}$ and $l^{u}$, respectively.

Substituting the expressions obtained in (24) and (28) for $\mathbb{E}\left(\tilde{\epsilon}_{1}^{R}\right)$ and $\mathbb{E}\left(\tilde{\epsilon}_{1}^{D_{i}}\right)$ respectively, in (18), yields the final 


$$
\begin{aligned}
\mathbb{E}\left(\tilde{\epsilon}_{1}^{R}\right) \approx & \frac{\tau_{1}^{R} \sqrt{n_{1}} a_{1} \beta_{S R}}{\Gamma\left(\alpha_{S R}\right) a_{2}}\left[\frac{\left(a_{1}-\zeta_{1}^{R} a_{2}\right)}{a_{1} \beta_{S R}} \bar{\Gamma}\left(\alpha_{S R}, \frac{\beta_{S R} \zeta_{1}^{R}}{\mu \rho_{S}\left(a_{1}-\zeta_{1}^{R} a_{2}\right)}\right)-\frac{\left(a_{1}-\Delta_{1}^{R} a_{2}\right)}{a_{1} \beta_{S R}}\right. \\
& \times \bar{\Gamma}\left(\alpha_{S R}, \frac{\beta_{S R} \Delta_{1}^{R}}{\mu \rho_{S}\left(a_{1}-\Delta_{1}^{R} a_{2}\right)}\right)+\frac{e^{\frac{\beta_{S R}}{\mu \rho_{S} a_{2}}}}{\left(\mu \rho_{S} a_{2}\right)^{\alpha_{S R}}} \times\left\{\sum_{u=0}^{\alpha_{S R}-1}\left(\begin{array}{c}
\alpha_{S R}-1 \\
u
\end{array}\right)\left(-\beta_{S R}\right)^{\alpha_{S R}-u-1}\left(\mu \rho_{S} a_{2}\right)^{u}\right. \\
& \left.\left.\times\left\{\bar{\Gamma}\left(u, \frac{\beta_{S R} a_{1}}{\mu \rho_{S} a_{2}\left(a_{1}-\Delta_{1}^{R} a_{2}\right)}\right)-\bar{\Gamma}\left(u, \frac{\beta_{S R} a_{1}}{\mu \rho_{S} a_{2}\left(a_{1}-\zeta_{1}^{R} a_{2}\right)}\right)\right\}\right\}\right] .
\end{aligned}
$$



$$
\begin{aligned}
& \mathbb{E}\left(\tilde{\epsilon}_{1}^{D_{i}}\right) \approx \frac{b_{1} \tau_{1}^{D_{i}} \sqrt{n_{2}}}{b_{2}} \sum_{v=0}^{\tilde{v}} \sum_{k=0}^{v} w_{v, k}^{D_{i}}\left\{\frac{\bar{\Gamma}\left(s_{k}^{D_{i}}, \sqrt{\frac{4 \zeta_{1}^{D_{i}} n_{2} \beta_{S R} \beta_{R D_{i}}}{n_{1} \eta \tilde{\mu}\left(b_{1}-\zeta_{1}^{D_{i}} b_{2}\right) \rho_{S}}}\right)}{\frac{b_{1}}{\left(b_{1}-\zeta_{1}^{D_{i}} b_{2}\right)}}-\frac{\bar{\Gamma}\left(s_{k}^{D_{i}}, \sqrt{\frac{4 \Delta_{1}^{D_{i}} n_{2} \beta_{S R} \beta_{R D_{i}}}{n_{1} \eta \tilde{\mu}\left(b_{1}-\Delta_{1}^{D_{i}} b_{2}\right) \rho_{S}}}\right)}{\frac{b_{1}}{\left(b_{1}-\Delta_{1}^{D_{i}} b_{2}\right)}}\right. \\
&\left.+\sum_{l=0}^{l^{u}}\left(\frac{4 n_{2} \beta_{S R} \beta_{R D_{i}}}{n_{1} \eta \tilde{\mu} b_{2} \rho_{S}}\right)^{\frac{l+s_{k}^{D_{i}}}{2}} \frac{(-1)^{l}}{l !} \hat{I}_{i, l}^{D_{i}}\right\},
\end{aligned}
$$

closed form expression of the average end-to-end BLER at user $D_{1}$. The average BLER for user $D_{2}$ is determined next.

Since, the expression of $\mathbb{E}\left(\tilde{\epsilon}_{1}^{R}\right)$ has been obtained in (24), while that of $\mathbb{E}\left(\tilde{\epsilon}_{1}^{D_{2}}\right)$ has been given in (28), to obtain $\mathbb{E}\left(\epsilon^{D_{2}}\right)$ from (19) we proceed to obtain $\mathbb{E}\left(\tilde{\epsilon}_{2}^{R}\right)$. Accordingly, we begin with the definition of $\gamma_{2}^{R}=a_{2} \mu \rho_{S}\left|h_{S R}\right|^{2}$ in (6), the CDF $F_{\gamma_{2}^{R}}(x)$, can be readily determined as $F_{\gamma_{2}^{R}}(x)=$ $\frac{1}{\Gamma\left(\alpha_{S R}\right)} \bar{\Gamma}\left(\alpha_{S R}, \frac{\beta_{S R} x}{\mu a_{2} \rho_{S}}\right)$. Substituting this in (22) leads to the expression of $\mathbb{E}\left(\tilde{\epsilon}_{2}^{R}\right)$ :

$$
\begin{aligned}
\mathbb{E}\left(\tilde{\epsilon}_{2}^{R}\right) & \approx \tau_{2}^{R} \sqrt{n_{1}} \int_{\zeta_{2}^{R}}^{\Delta_{2}^{R}} F_{\gamma_{2}^{R}}(x) d x \\
& =\frac{\tau_{2}^{R} \sqrt{n_{1}}}{\Gamma\left(\alpha_{S R}\right)} \int_{\zeta_{2}^{R}}^{\Delta_{2}^{R}} \bar{\Gamma}\left(\alpha_{S R}, \frac{\beta_{S R} x}{\mu a_{2} \rho_{S}}\right) d x .
\end{aligned}
$$

Using the substitution $\frac{x \beta_{S R}}{\mu a_{2} \rho_{S}}=t$, the expression in (29) can be simplified using integration by parts and upon invoking the identity $\frac{d \bar{\Gamma}(s, x)}{d x}=x^{s-1} e^{-x}$, to obtain the expression of $\mathbb{E}\left(\tilde{\epsilon}_{2}^{R}\right)$ as given below:

$$
\begin{aligned}
\mathbb{E} & \left(\tilde{\epsilon}_{2}^{R}\right) \approx \frac{\tau_{2}^{R} \sqrt{n_{1}} \mu a_{2} \rho_{S}}{\Gamma\left(\alpha_{S R}\right) \beta_{S R}}\left\{\frac{\Delta_{2}^{R} \beta_{S R}}{\mu a_{2} \rho_{S}} \bar{\Gamma}\left(\alpha_{S R}, \frac{\Delta_{2}^{R} \beta_{S R}}{\mu a_{2} \rho_{S}}\right)\right. \\
& -\frac{\zeta_{2}^{R} \beta_{S R}}{\mu a_{2} \rho_{S}} \bar{\Gamma}\left(\alpha_{S R}, \frac{\zeta_{2}^{R} \beta_{S R}}{\mu a_{2} \rho_{S}}\right)+\bar{\Gamma}\left(\alpha_{S R}+1, \frac{\zeta_{2}^{R} \beta_{S R}}{\mu a_{2} \rho_{S}}\right) \\
& \left.-\bar{\Gamma}\left(\alpha_{S R}+1, \frac{\Delta_{2}^{R} \beta_{S R}}{a_{2} \rho_{S}}\right)\right\} .
\end{aligned}
$$

To obtain the only remaining BLER term $\mathbb{E}\left(\tilde{\epsilon}_{2}^{D_{2}}\right)$ in (19), one can start with the SNR expression of $\gamma_{2}^{D_{2}}=\frac{b_{2} P_{R}\left|h_{R D_{2}}\right|^{2}}{\sigma^{2}}=$ $\frac{b_{2} \eta \tilde{\mu} n_{1} \rho_{S}\left|h_{S R}\right|^{2}\left|h_{R D_{2}}\right|^{2}}{n_{1}}$ as given in (10). Employing the result in Theorem 1, the corresponding CDF for the same can be formulated as

$$
F_{\gamma_{2}^{D_{2}}}(x) \approx \sum_{v=0}^{\tilde{v}} \sum_{k=0}^{v} w_{v, k}^{D_{2}} \bar{\Gamma}\left(s_{k}^{D_{2}}, \sqrt{\frac{x \Upsilon_{2}}{b_{2} \rho_{S}}}\right) d x,
$$

where the above expression is tight even for small values of $\tilde{v}$ and the quantity $\Upsilon_{2}=\frac{4 n_{2} \beta_{S R} \beta_{R D_{2}}}{\eta \tilde{\mu} n_{1}}$. Substituting the above result for $F_{\gamma_{2} D_{2}}(x)$ in (22) followed by the further simplifications in Section IV of our technical report in [63] leads to the expression of $\mathbb{E}\left(\tilde{\epsilon}_{2}^{D_{2}}\right)$ as given in (32).

Substituting the expressions obtained in (24), (28), (30) and (32), in (19), yields the final closed-form expression for the average end-to-end BLER $\mathbb{E}\left(\epsilon^{D_{2}}\right)$ at user $D_{2}$.

\section{HIGH SNR BLER, DIVERSITY ORDER AND INTERFERENCE ANALYSIS}

\section{A. Average BLER and diversity order at users $D_{1}, D_{2}$}

The asymptotic BLER expressions at high SNRs that is when $\rho_{S} \rightarrow \infty$, will be determined for the different users below, which yield further insights into the performance of the SWIPT-based FBL non-orthogonal systems. The results below pertaining to the BLER of decoding $x_{1}$ at terminal $U, U \in\left\{R, D_{1}, D_{2}\right\}$ and to the BLER of decoding $x_{2}$ at $\tilde{U}, \tilde{U} \in\left\{R, D_{2}\right\}$ are used in the subsequent analysis. 


$$
\begin{aligned}
\mathbb{E}\left(\tilde{\epsilon}_{2}^{D_{2}}\right) & \approx \frac{b_{2} \rho_{S} \tau_{2}^{D_{2}} \sqrt{n_{2}}}{\Upsilon_{2}} \sum_{v=0}^{\tilde{v}} \sum_{k=0}^{v} w_{v, k}^{D_{2}}\left\{\frac{\Upsilon_{2} \Delta_{2}^{D_{2}}}{b_{2} \rho_{S}} \bar{\Gamma}\left(s_{k}^{D_{2}}, \sqrt{\frac{\Upsilon_{2} \Delta_{2}^{D_{2}}}{b_{2} \rho_{S}}}\right)-\frac{\Upsilon_{2} \zeta_{2}^{D_{2}}}{b_{2} \rho_{S}}\right. \\
& \left.\times \bar{\Gamma}\left(s_{k}^{D_{2}}, \sqrt{\frac{\Upsilon_{2} \zeta_{2}^{D_{2}}}{b_{2} \rho_{S}}}\right)+\Gamma\left(s_{k}^{D_{2}}+2, \sqrt{\frac{\Upsilon_{2} \Delta_{2}^{D_{2}}}{b_{2} \rho_{S}}}\right)-\Gamma\left(s_{k}^{D_{2}}+2, \sqrt{\frac{\Upsilon_{2} \zeta_{2}^{D_{2}}}{b_{2} \rho_{S}}}\right)\right\} .
\end{aligned}
$$

Lemma 1. At high SNR, the average BLER of decoding $x_{1}$ at terminal $U$, can be obtained as

$$
\begin{aligned}
\mathbb{E}\left(\bar{\epsilon}_{1}^{U}\right)= & \frac{\bar{c}^{U} \tau_{1}^{U} \sqrt{n_{j}}}{\alpha_{e}\left(\alpha_{e}+1\right)}\left(\frac{\beta_{e}}{\mu_{e} \times a_{1, e} \times \rho_{S}}\right)^{\alpha_{e}} \\
\times & \left\{f_{0}\left(a_{1, e}, a_{2, e}, \Delta_{1}^{U}, \alpha_{e}\right)\right. \\
& \left.\quad-f_{0}\left(a_{1, e}, a_{2, e}, \zeta_{1}^{U}, \alpha_{e}\right)\right\},
\end{aligned}
$$

where we have $f_{0}(w, x, y, z)=y^{z+1} \times$ ${ }_{2} F_{1}\left(z, z+1 ; z+2 ; \frac{y x}{w}\right)$ and the term ${ }_{2} F_{1}\left(x_{1}, x_{2} ; x_{3} ; x_{4}\right)$ represents the Gaussian geometric function defined as [66]:

$$
{ }_{2} F_{1}\left(x_{1}, x_{2} ; x_{3} ; x_{4}\right)=\sum_{v=0}^{\infty} \frac{\left(x_{1}\right)_{v}\left(x_{2}\right)_{v}}{\left(x_{3}\right)_{v}} \times \frac{x_{4}^{v}}{v !},
$$

where $(q)_{v}=q \times(q+1) \cdots \times(q+v-1)$ denotes the Pochhammer symbol [66] and the other terms $\bar{c}^{U}, \alpha_{e}, \beta_{e}, \mu_{e}, a_{1, e}, a_{2, e}$ are defined in (35) and (36).

Proof. A detailed proof is given in Section V of our technical report in [63].

Lemma 2. At high SNR, the average BLER of decoding $x_{2}$ at terminal $\tilde{U} \in\left\{R, D_{2}\right\}$, given that $x_{1}$ has already been correctly decoded at the terminal, can be written as

$$
\mathbb{E}\left(\bar{\epsilon}_{2}^{\tilde{U}}\right)=\tilde{c} \tilde{U} \sum_{t=0,2,4, \cdots}^{\alpha_{e}} \frac{\left(\begin{array}{c}
\alpha_{e}+1 \\
t+1
\end{array}\right)}{\left(\tau_{2}^{\tilde{U}}\right)^{t}},
$$

where $\tau_{2}^{\tilde{U}}=\frac{1}{2 \psi_{2}^{\tilde{U}} \tau_{2}^{\tilde{U}} \sqrt{n_{j}}}, \tilde{c}^{\tilde{U}}=\frac{\bar{c}^{\tilde{U}}}{\alpha_{e}\left(\alpha_{e}+1\right)}\left(\frac{\beta_{e} \psi_{2}^{\tilde{U}}}{\mu_{e} \times a_{2, e} \times \rho_{S}}\right)^{\alpha_{e}}$ and the terms $\overline{\bar{U}}, \alpha_{e}, \beta_{e}, \mu_{e}, a_{2, e}, n_{j}$ are defined in (35), (36).

Proof. A detailed proof is given in Section VI of our technical report in [63].

The asymptotic BLERs at high SNRs for decoding $x_{i}$ at $D_{i}$ are now derived below.

Proposition 1. At high SNR, the analytical expressions for the end-to-end BLER at users $D_{1}, D_{2}$ can be formulated as

$$
\begin{aligned}
& \mathbb{E}\left(\bar{\epsilon}^{D_{1}}\right) \approx \mathbb{E}\left(\bar{\epsilon}_{1}^{R}\right)+\mathbb{E}\left(\bar{\epsilon}_{1}^{D_{1}}\right), \\
& \mathbb{E}\left(\bar{\epsilon}^{D_{2}}\right) \approx \mathbb{E}\left(\bar{\epsilon}_{1}^{R}\right)+\mathbb{E}\left(\bar{\epsilon}_{2}^{R}\right)+\mathbb{E}\left(\bar{\epsilon}_{1}^{D_{2}}\right)+\mathbb{E}\left(\bar{\epsilon}_{2}^{D_{2}}\right) .
\end{aligned}
$$

Proof. This result follows using the expressions for $\mathbb{E}\left(\epsilon^{D_{1}}\right), \mathbb{E}\left(\epsilon^{D_{2}}\right)$ from (18),(19) respectively, followed by substituting the results in Lemma 1 and Lemma 2 for the various quantities.

The following results now characterize the diversity order of the BLER at users 1,2.
Remark 1. At high SNR, the average end-to-end BLER at user $D_{i}$, where $i \in\{1,2\}$, varies as $\mathbb{E}\left(\bar{\epsilon}^{D_{i}}\right) \propto \rho_{S}-D O^{i}$, where the diversity order is given by

$$
D O^{i}= \begin{cases}\min \left(\alpha_{S R}, \alpha_{R D_{i}}\right), & \alpha_{S R} \neq \alpha_{R D_{i}} \\ \alpha_{S R}-1, & \text { otherwise. }\end{cases}
$$

\section{B. Impact of interference on BLER}

Let $\mathbb{E}\left(\bar{\epsilon}_{x_{1} \nrightarrow x_{2}}^{U}\right)$ denote the average BLER of decoding $x_{1}$ without any interference from $x_{2}$ at terminal $U$. Similarly, let $\mathbb{E}\left(\bar{\epsilon}_{x_{1} \rightarrow x_{2}}^{U}\right)$ denote the average BLER contribution arising due to the interference imposed by the superposition of $x_{2}$, while decoding $x_{1}$ at terminal $U$. The next result presents additional insights regarding the interference imposed by $x_{2}$ on the BLER while decoding symbol $x_{1}$ at the terminal $U, U \in\left\{R, D_{1}, D_{2}\right\}$ in our FBL non-orthogonal system.

Theorem 2. At high SNR, the average BLER contribution during the decoding of $x_{1}$ arising due to the interference caused by the superposition of symbol $x_{2}$ at node $U$ is upper bounded as

$$
\begin{aligned}
\mathbb{E}\left(\bar{\epsilon}_{x_{1} \rightarrow x_{2}}^{U}\right) \leq & \frac{\bar{c}^{U} \tau_{1}^{U} \sqrt{n_{j}}}{\left(\alpha_{e}+2\right)}\left(\frac{\beta_{e}}{\mu_{e} \times a_{1, e} \times \rho_{S}}\right)^{\alpha_{e}} \frac{a_{2, e}}{a_{1, e}} \\
\times & \left\{f_{0}\left(a_{1, e}, a_{2, e}, \Delta_{1}^{U}, \alpha_{e}+1\right)\right. \\
& \left.\quad-f_{0}\left(a_{1, e}, a_{2, e}, \zeta_{1}^{U}, \alpha_{e}+1\right)\right\},
\end{aligned}
$$

where the terms $\bar{c}^{U}, \alpha_{e}, \beta_{e}, \mu_{e}, a_{1, e}, a_{2, e}, n_{j}, f_{0}(\cdot, \cdot, \cdot, \cdot)$ are defined in (33), (35), (36).

Proof. A detailed proof is given in Section VII of our technical report in [63].

Remark 2. At high SNR, the BLER contribution due to the interference arising from the superposition of symbols $x_{1}$ and $x_{2}$ imposed on the end-to-end BLER at user $D_{i}$, which is denoted by $\mathbb{E}\left(\bar{\epsilon}_{\text {inf }}^{D_{i}}\right), i \in\{1,2\}$, is upper bounded as follows:

$$
\mathbb{E}\left(\bar{\epsilon}_{i n f}^{D_{i}}\right) \leq \mathbb{E}\left(\bar{\epsilon}_{x_{1} \rightarrow x_{2}}^{R}\right)+\mathbb{E}\left(\bar{\epsilon}_{x_{1} \rightarrow x_{2}}^{D_{i}}\right) .
$$

Hence, the net average BLER at $D_{1}, D_{2}$ can be re-written in a more insightful manner as:

$$
\begin{aligned}
\mathbb{E}\left(\bar{\epsilon}^{D_{1}}\right) \leq & \mathbb{E}\left(\bar{\epsilon}_{x_{1} \dashv x_{2}}^{R}\right)+\mathbb{E}\left(\bar{\epsilon}_{x_{1} \dashv x_{2}}^{D_{1}}\right)+\mathbb{E}\left(\bar{\epsilon}_{\text {inf }}^{D_{1}}\right), \\
\mathbb{E}\left(\bar{\epsilon}^{D_{2}}\right) \leq & \mathbb{E}\left(\bar{\epsilon}_{x_{1} \nrightarrow x_{2}}^{R}\right)+\mathbb{E}\left(\bar{\epsilon}_{2}^{R}\right) \\
& +\mathbb{E}\left(\bar{\epsilon}_{x_{1} \dashv x_{2}}^{D_{2}}\right)+\mathbb{E}\left(\bar{\epsilon}_{2}^{D_{2}}\right)+\mathbb{E}\left(\bar{\epsilon}_{\text {inf }}^{D_{2}}\right),
\end{aligned}
$$

where $\mathbb{E}\left(\bar{\epsilon}_{x_{1} \nrightarrow x_{2}}^{R}\right)$ denotes the average BLER of decoding $x_{1}$ without any interference from $x_{2}$ at relay $R, \mathbb{E}\left(\bar{\epsilon}_{x_{1} \nrightarrow x_{2}}^{D_{i}}\right)$ 


$$
\begin{aligned}
& \left(\alpha_{e} ; \beta_{e} ; \mu_{e} ; a_{1, e} ; a_{2, e}\right)= \begin{cases}\left(\alpha_{S R} ; \beta_{S R} ; \mu ; a_{1} ; a_{2}\right), & U=\{R\} \\
\left(s_{0}^{D_{i}} ; \alpha_{S R} \beta_{R D_{i}} ; \frac{\eta \tilde{\mu} n_{1}}{4 n_{2}} ; b_{1} ; b_{2}\right), & U \in\left\{D_{i}\right\},\end{cases} \\
& \bar{c}^{U}=\left\{\begin{array}{l}
\frac{1}{\Gamma\left(\alpha_{e}\right)}, U \in\{R\} \\
\frac{\sqrt{\pi} \Gamma\left(2\left|\alpha_{S R}-\alpha_{R D_{i}}\right|\right)}{2^{2 \alpha_{S R}+2 \alpha_{R D_{i}}-1} \Gamma\left(\alpha_{S R}\right) \Gamma\left(\alpha_{R D_{i}}\right) \Gamma\left(\frac{1}{2}-\left|\alpha_{S R}+\alpha_{R D_{i}}\right|\right)}, U \in\left\{D_{i}\right\},
\end{array} \quad j=\left\{\begin{array}{l}
1, U \in\{R\}, \\
2, U \in\left\{D_{i}\right\} .
\end{array}\right.\right.
\end{aligned}
$$

represents the average BLER of decoding $x_{1}$ without any interference from $x_{2}$ at users $D_{i}, i=1,2$ and $\mathbb{E}\left(\bar{\epsilon}_{i n f}^{D_{i}}\right)$ denotes the end-to-end average BLER contribution arising due to the interference from $x_{2}$, while decoding $x_{1}$ at user $D_{i}$.

\section{JOINT POWER, SPLITTING FACTOR AND BLOCKLENGTH OPTIMIZATION}

In $5 \mathrm{G}$ wireless networks of the future, many of the communication devices will find applications in IoT and realtime machine-to-machine communications [7], [25]. This will require systems with low communication latency, high decoding reliability and energy efficiency [1], [3]. Therefore, the focus of this section is to develop a framework of determining the optimal source, relay power allocation and relay splitting factor for the minimization of total blocklength in our energy harvesting FBL non-orthogonal system under strict reliability constraints. Accordingly, the optimization problem of blocklength minimization can be framed as

$$
\underset{a_{1}, a_{2}, b_{1}, b_{2}, \mu, \tilde{\mu}, n_{1}, n_{2}}{\operatorname{minimize}} n_{1}+n_{2}
$$

subject to

$$
\begin{aligned}
& \mathbb{E}\left(\bar{\epsilon}^{D_{1}}\right) \approx \mathbb{E}\left(\bar{\epsilon}_{1}^{R}\right)+\mathbb{E}\left(\bar{\epsilon}_{1}^{D_{1}}\right) \leq \epsilon_{1}^{t h}, \\
& \mathbb{E}\left(\bar{\epsilon}^{D_{2}}\right) \approx \sum_{j=1}^{2} \mathbb{E}\left(\bar{\epsilon}_{j}^{R}\right)+\mathbb{E}\left(\bar{\epsilon}_{j}^{D_{2}}\right) \leq \epsilon_{2}^{t h}, \\
& n_{1} \geq 100, n_{2} \geq 100, \mu>0, \tilde{\mu}>0, \mu+\tilde{\mu} \leq 1, \\
& a_{1} \geq a_{2}>0, b_{1} \geq b_{2}>0, a_{1}+a_{2}=1, b_{1}+b_{2}=1,
\end{aligned}
$$

where the objective function (OF) $n_{1}+n_{2}$ denotes the total blocklength and the reliability or QoS constraints are given by restricting the net end-to-end BLERs $\mathbb{E}\left(\bar{\epsilon}^{D_{1}}\right), \mathbb{E}\left(\bar{\epsilon}^{D_{2}}\right)$, for users 1,2 to be lower than $\epsilon_{1}^{t h}, \epsilon_{2}^{t h}$ respectively. The constraints $a_{1} \geq a_{2}$ and $b_{1} \geq b_{2}$ ensure that a higher power is allocated to the user with a lower average channel gain [59] and guarantees that the SIC decoding order is not changed [67]. Furthermore, $n_{1}, n_{2} \geq 100$ denotes the minimum blocklength constraints to be satisfied for the results in (11) to be tight [22], and consequently are required for the BLER expressions of user $D_{1}, D_{2}$ in Section III and IV to be accurate. It can be seen that the optimization problem in (43) is non-convex and mathematically intractable. However, using the procedure described below, it can be transformed into a geometric program (GP) and solved efficiently. Accordingly, at high SNR, our optimization problem of blocklength minimization as given in (43) above be written as given in (44), where the inequality constraints in $(44 b),(44 c)$ follow by using the result in [68], which states that the average BLER can be approximated by the outage probability as

$$
\mathbb{E}(\bar{\epsilon}) \approx F_{\gamma}\left(2^{\frac{N}{n}}-1\right),
$$

followed by using the high SNR approximation of $\bar{\Gamma}(s, x) \approx$ $\frac{x^{s}}{s}, x \ll 1, s>0$, in the expressions for the CDFs $F_{\gamma_{1}^{R}}\left(\psi_{1}^{R}\right), F_{\gamma_{2}^{R}}\left(\psi_{2}^{R}\right), F_{\gamma_{1}^{D_{i}}}\left(\psi_{1}^{D_{i}}\right), F_{\gamma_{2}^{D_{2}}}\left(\psi_{2}^{D_{2}}\right)$. To further simplify (44), we proceed by re-writing the inequality constraint in $(44 \mathrm{~b})$ as

$$
\begin{aligned}
(44 \mathrm{~b}) & \equiv \sum_{i=1}^{2} f_{i}(\underline{\xi}) \leq \epsilon_{1}^{t h} \\
& \equiv\left\{f_{i}(\underline{\xi}) \leq t_{i}, 1 \leq i \leq 2, \sum_{i=1}^{2} t_{i} \leq \epsilon_{1}^{t h}\right\},
\end{aligned}
$$

where $\underline{\xi}=\left[a_{2}, b_{2}, n_{1}, n_{2}, \mu, \tilde{\mu}, \psi_{1}^{U}, \psi_{2}^{\tilde{U}}, \tau_{1}^{U}, \tau_{2}^{\tilde{U}}, \Delta_{1}^{U}, \Delta_{2}^{\tilde{U}}\right]^{T}$, $U \in\left\{R, D_{1}, D_{2}\right\}, \tilde{U} \in\left\{R, D_{2}\right\}$ and the individual functions $f_{i}(\underline{\xi}), 1 \leq i \leq 2$, are given as

$$
\begin{aligned}
& f_{1}(\underline{\xi})=c_{1}\left(\frac{\psi_{1}^{R}}{\mu\left(1-a_{2}\left(1+\psi_{1}^{R}\right)\right)}\right)^{\alpha_{S R}}, \\
& f_{2}(\underline{\xi})=d_{1}\left(\frac{n_{2} \psi_{1}^{D_{1}}}{\tilde{\mu} n_{1}\left(1-b_{2}\left(1+\psi_{1}^{D_{1}}\right)\right)}\right)^{\frac{s_{0}^{D_{1}}}{2}},
\end{aligned}
$$

where the constants $c_{1}=\frac{\left(\frac{\beta_{S R}}{\rho_{S}}\right)^{\alpha_{S R}}}{\Gamma\left(\alpha_{S R}\right) \alpha_{S R}}$ and $d_{1}=$ $\frac{\left(\sum_{v=0}^{\tilde{v}} w_{v, 0}^{D_{1}}\right)}{s_{0}^{D_{1}}}\left(\frac{4 \beta_{S R} \beta_{R D_{1}}}{\eta \rho_{S}}\right)^{\frac{s_{0}^{D_{1}}}{2}}$. Thus, the inequality constraints in (46) can equivalently be written as

$$
\begin{gathered}
f_{1}(\underline{\xi}) \leq t_{1} \equiv \psi_{1}^{R}\left(a_{2}+\mu^{-1} c_{1}^{\frac{1}{\alpha_{S R}}} t_{1}^{\frac{-1}{\alpha_{S R}}}\right)+a_{2} \leq 1 \\
f_{2}(\underline{\xi}) \leq t_{2} \equiv \psi_{1}^{D_{1}}\left(b_{2}+\tilde{\mu}^{-1} n_{1}^{-1} n_{2}\left(d_{1}\right)^{\frac{2}{s_{0}}}\left(t_{2}\right)^{\frac{-2}{s_{0}}}\right) \\
\quad+b_{2} \leq 1 \\
\left(\epsilon_{1}^{t h}\right)^{-1}\left(t_{1}+t_{2}\right) \leq 1 .
\end{gathered}
$$

Using the Taylor series expansions $2^{x} \approx$ $\sum_{v=0}^{v_{\max }} \frac{(\ln 2 \times x)^{v}}{v !}, 4^{x} \approx \sum_{v=0}^{v_{\max }} \frac{(\ln 4 \times x)^{v}}{v !}$, where the constant $v_{\max }$ denotes the upper limit of the summation which can be set suitably, the quantities $\psi_{1}^{R}, \psi_{1}^{D_{1}}$ in (48) can be approximated as

$\psi_{1}^{R} \approx \sum_{v=1}^{v_{\max }}\left(\ln 2 \frac{N_{1}}{n_{1}}\right)^{v} \frac{1}{v !}, \psi_{1}^{D_{1}} \approx \sum_{v=1}^{v_{\max }}\left(\ln 2 \frac{N_{1}}{n_{2}}\right)^{v} \frac{1}{v !}$. 
$\underset{a_{1}, a_{2}, b_{1}, b_{2}, \mu, \tilde{\mu}, n_{1}, n_{2}}{\operatorname{minimize}} n_{1}+n_{2}$

subject to $\frac{1}{\Gamma\left(\alpha_{S R}\right) \alpha_{S R}}\left(\frac{\beta_{S R} \psi_{1}^{R}}{\mu \rho_{S}\left(a_{1}-\psi_{1}^{R} a_{2}\right)}\right)^{\alpha_{S R}}+\frac{1}{s_{0}^{D_{1}}}\left(\frac{4 n_{2} \beta_{S R} \beta_{R D_{1}} \psi_{1}^{D_{1}}}{\eta \tilde{\mu} n_{1} \rho_{S}\left(b_{1}-\psi_{1}^{D_{1}} b_{2}\right)}\right)^{\frac{s_{0}^{D_{1}}}{2}}\left(\sum_{v=0}^{\tilde{v}} w_{v, 0}^{D_{1}}\right) \leq \epsilon_{1}^{t h}$,

$$
\begin{aligned}
& \frac{1}{\Gamma\left(\alpha_{S R}\right) \alpha_{S R}}\left(\frac{\beta_{S R} \psi_{1}^{R}}{\mu \rho_{S}\left(a_{1}-\psi_{1}^{R} a_{2}\right)}\right)^{\alpha_{S R}}+\frac{1}{\Gamma\left(\alpha_{S R}\right) \alpha_{S R}}\left(\frac{\beta_{S R} \psi_{2}^{R}}{\mu a_{2} \rho_{S}}\right)^{\alpha_{S R}} \\
& +\frac{\left(\sum_{v=0}^{\tilde{v}} w_{v, 0}^{D_{2}}\right)}{s_{0}^{D_{2}}}\left\{\left(\frac{4 n_{2} \beta_{S R} \beta_{R D_{2}} \psi_{1}^{D_{2}}}{\eta \tilde{\mu} n_{1} \rho_{S}\left(b_{1}-\psi_{1}^{D_{2}}\right)}\right)^{\frac{s_{0}^{D_{2}}}{2}}+\left(\frac{4 n_{2} \beta_{S R} \beta_{R D_{1}} \psi_{2}^{D_{2}}}{b_{2} n_{1} \eta \tilde{\mu} \rho_{S}}\right)^{\frac{s_{0}^{D_{2}}}{2}}\right\} \leq \epsilon_{2}^{t h},
\end{aligned}
$$

$a_{1} \geq a_{2}>0, b_{1} \geq b_{2}>0, a_{1}+a_{2}=1, b_{1}+b_{2}=1$,

$n_{1} \geq 100, n_{2} \geq 100, \mu>0, \tilde{\mu}>0, \mu+\tilde{\mu} \leq 1$,

By employing the above approximations, the inequality constraints in (48) can be approximated as shown below:

$$
\begin{aligned}
& f_{1}(\underline{\xi}) \leq t_{1} \cong\left(\sum_{v=1}^{v_{\max }}\left(\left(\ln 2 \frac{N_{1}}{n_{1}}\right)^{v} \frac{1}{v !}\right)\right) \\
& \times\left(a_{2}+\mu^{-1} c_{1}^{\frac{1}{\alpha_{S R}}} t_{1}^{\frac{-1}{\alpha_{S R}}}\right)+a_{2} \leq 1, \\
& f_{2}(\underline{\xi}) \leq t_{2} \cong\left(\sum_{v=1}^{v_{\max }}\left(\left(\ln 2 \frac{N_{1}}{n_{2}}\right)^{v} \frac{1}{v !}\right)\right) \\
& \times\left(b_{2}+\tilde{\mu}^{-1} n_{1}^{-1} n_{2}\left(d_{1}\right)^{\frac{2}{s_{0}}}\left(t_{2}\right)^{\frac{-2}{D_{0}^{D}}}\right)+b_{2} \leq 1, \\
&\left(\epsilon_{1}^{t h}\right)^{-1}\left(t_{1}+t_{2}\right) \leq 1 .
\end{aligned}
$$

Thus, the inequality constraint in (44b) can be approximately represented by a set of multiple posynomial [69] inequality constraints that is $(44 b) \cong[(50 a),(50 b),(50 c)]$.

Similarly, the other inequality constraint in (44c) can be decomposed in terms of multiple posynomial inequality constraints such that $(44 \mathrm{c}) \cong[(51 \mathrm{a}),(51 \mathrm{~b}),(51 \mathrm{c}),(51 \mathrm{~d}),(51 \mathrm{e})]$ where

$$
\begin{aligned}
& \left(\sum_{v=1}^{v_{\max }}\left(\left(\ln 2 \frac{N_{1}}{n_{1}}\right)^{v} \frac{1}{v !}\right)\right) \\
& \times\left(a_{2}+\mu^{-1} c_{1}^{\frac{1}{\alpha_{S R}}} t_{3}^{\frac{-1}{\alpha_{S R}}}\right)+a_{2} \leq 1, \\
& \left(\sum_{v=1}^{v_{\max }}\left(\left(\ln 2 \frac{N_{1}}{n_{2}}\right)^{v} \frac{1}{v !}\right)\right) \\
& \times\left(b_{2}+\tilde{\mu}^{-1} n_{1}^{-1} n_{2}\left(d_{2}\right)^{\frac{2}{s_{0}}}\left(t_{4}\right)\left(\frac{-2}{s_{0}}\right)\right)+b_{2} \leq 1, \\
& \left.\left(\sum_{v=1}^{v_{\max }}\left(\left(\ln 2 \frac{N_{2}}{n_{1}}\right)^{v} \frac{1}{v !}\right)\right){ }^{\frac{1}{D_{2}}}\right) \\
& \times\left(\mu^{-1} a_{2}^{-1} c_{1}^{\frac{1}{\alpha_{S R}}} t_{5}^{\frac{-1}{\alpha_{S R}}}\right) \leq 1
\end{aligned}
$$

$$
\begin{aligned}
& \left(\sum_{v=1}^{v_{\max }}\left(\left(\ln 2 \frac{N_{2}}{n_{2}}\right)^{v} \frac{1}{v !}\right)\right) \\
& \times\left(\tilde{\mu}^{-1} b_{2}^{-1} n_{1}^{-1} n_{2} d_{2}^{\frac{2}{s_{0}}} \frac{-2}{t_{6}^{s_{2}}}\right) \leq 1, \\
& \left(\epsilon_{2}^{t h}\right)^{-1}\left(t_{3}+t_{4}+t_{5}+t_{6}\right) \leq 1,
\end{aligned}
$$

where $d_{2}=\frac{\left(\sum_{v=0}^{\tilde{v}} w_{v, 0}^{D_{2}}\right)}{s^{D_{2}}}\left(\frac{4 \beta_{S R} \beta_{R D_{2}}}{\eta \rho_{S}}\right)^{\frac{s_{0}^{D_{2}}}{2}}$. This yields the approximate equivalent form of the optimization problem in (44) or (43) for the end-to-end blocklength minimization as shown below in (52).

$$
\underset{a_{2}, b_{2}, n_{1}, n_{2}, \mu, \tilde{\mu}, \underline{\mathbf{t}} \succ \underline{\mathbf{0}}}{\operatorname{minimize}} n_{1}+n_{2}
$$

subject to: $\quad(50 \mathrm{a})-(50 \mathrm{c}),(51 \mathrm{a})-(51 \mathrm{e}),(44 \mathrm{e}),(52 \mathrm{~b})$

$$
0<a_{2} \leq \frac{1}{2}, 0<b_{2} \leq \frac{1}{2}
$$

where $\underline{\mathbf{t}}=\left[t_{1}, t_{2}, t_{3}, t_{4}, t_{5}, t_{6}\right]^{T}$. The optimization problem in (52) is a GP, since the objective function is linear and the constraints are given by the sum of posynomial terms in $a_{2}, b_{2}, n_{1}, n_{2}, \mu, \tilde{\mu}, \underline{\mathbf{t}}$ [69]. Hence, it is a convex optimization problem. Our simulation results are presented next.

\section{Vi. Simulation Results}

TABLE II: Simulation Parameters

\begin{tabular}{|l|c|}
\hline Description: Parameter & Value \\
\hline Shape parameters: $\alpha_{S R}, \alpha_{R D_{1}}, \alpha_{R D_{2}}$ & $3,2,2$ \\
\hline Average channel gains: $\lambda_{S R}, \lambda_{R D_{1}}, \lambda_{R D_{2}}$ & $5,1,3$ \\
\hline Max. acceptable BLERs at $D_{1}, D_{2}: \epsilon_{1}^{t h}, \epsilon_{2}^{t h}$ & $10^{-5}, 10^{-4}$ \\
\hline Energy conversion efficiency [40]: $\eta$ & 0.5 \\
\hline Splitting factor at relay: $\mu$ & 0.5 \\
\hline Summation upper limits: $\tilde{v}, v_{\max }, l^{u}$ & $4,20,50$ \\
\hline Power factors at $S: a_{1}, a_{2}$ & $0.8,0.2$ \\
\hline Power factors at $\left.R: b_{1}, b_{2}\right)$ & $0.8,0.2$ \\
\hline No. of bits for $D_{1}, D_{2}: N_{1}, N_{2}$ & 50,50 \\
\hline No. of channel uses in Phase $1,2: n_{1}, n_{2}$ & 100,100 \\
\hline
\end{tabular}




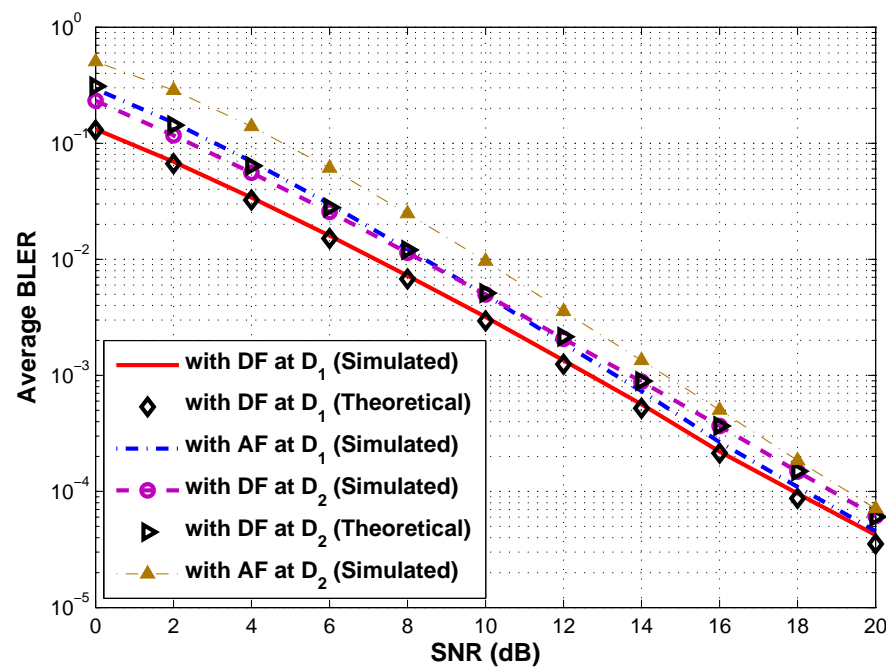

(a)

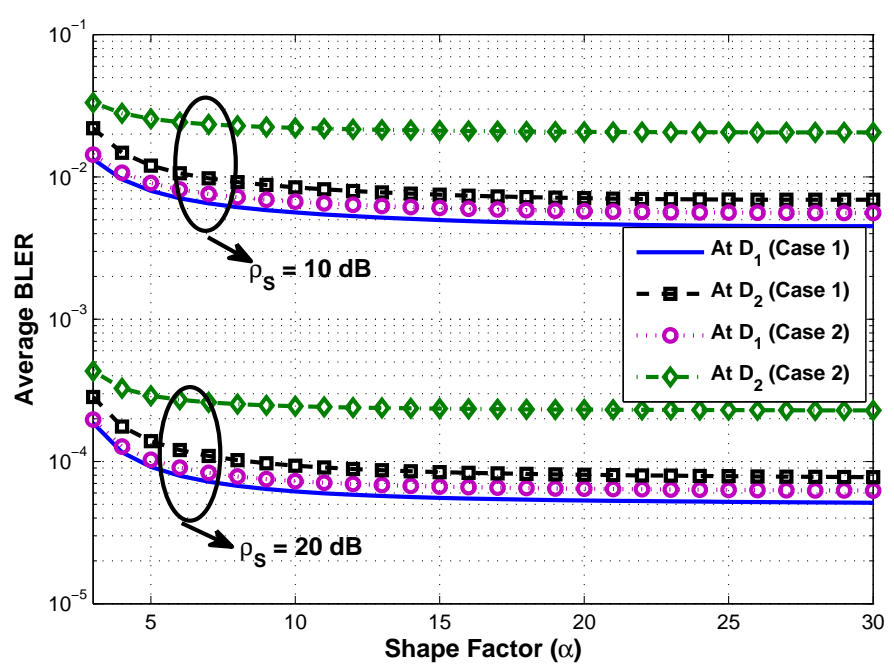

(b)

Fig. 2: (a) Average BLER versus SNR performance at $D_{1}, D_{2}$ (Eq. (18),(19)). (b) Average BLER versus shape factors $\alpha_{S R}, \alpha_{R D_{1}}, \alpha_{R D_{2}}$ performance at $D_{1}, D_{2}$ (Eq. (18),(19))

The simulation parameters have been set as in Table II, with some variations for the individual results that are explicitly stated later. The channel coefficients $h_{S R}, h_{R D_{i}}$ are Nakagami- $m$ distributed associated with the fading parameters $m_{S R}, m_{R D_{i}}$, respectively and average gains of $\mathbb{E}\left(\left|h_{S R}\right|^{2}\right)=$ $\lambda_{S R}, \mathbb{E}\left(\left|h_{R D_{i}}\right|^{2}\right)=\lambda_{R D_{i}}$. Therefore, the channel gains $\left|h_{Y}\right|^{2}, Y \in\left\{S R, R D_{i}\right\}$ are Gamma distributed with the shape and scale parameters $\alpha_{X}=m_{X}$ and $\beta_{X}=\frac{\alpha_{X}}{\lambda_{X}}$. Furthermore, since no mobility is considered, the path loss is fixed to the average gains of $\mathbb{E}\left(\left|h_{S R}\right|^{2}\right)=\lambda_{S R}, \mathbb{E}\left(\left|h_{R D_{i}}\right|^{2}\right)=\lambda_{R D_{i}}$ representing the effect of path loss. As described in Section II, the power factors $a_{i}, b_{i}$ represent the fraction of transmit power assigned to destination $D_{i}$ by $S$ and $R$, respectively.

Fig. 2(a) shows the average BLER versus SNR of $D_{1}, D_{2}$ for source and relay power factors $a_{1}=b_{1}=0.8$ and $n_{1}=n_{2}=200$. A close overlap can be observed between the simulated BLER values and the corresponding analytical values of (18),(19) obtained from the average BLER expressions derived for the two users in Section III, which highlight the validity of the analytical expressions and also justify the approximations employed in our BLER analysis. Moreover, for comparison, the average BLER performance of an AF relayaided similar non-orthogonal system has also been included in this figure. It can be observed that the proposed DF relay-aided system outperforms the one with an AF relay since, the later leads to significant noise amplification at the destination users $D_{1}, D_{2}$. Furthermore, BLER performance comparisons with the AF protocol, taking into account also the power associated with SIC at the relay, are presented in Figs. 3, 4(a) and 4(b) in Section X of our technical report in [63].

Fig. 2(b) demonstrates the impact of shape factors $\alpha_{S R}$ and $\alpha_{R D_{i}}$ on the average BLER performance of $D_{1}, D_{2}$. The parameters have been set as $n_{1}=n_{2}=100, \lambda_{S R}=$ $5, \lambda_{R D_{1}}=1, \lambda_{R D_{2}}=3$. Case 1 presents the variation of the average BLERs versus $\alpha_{S R}$ along with $\alpha_{R D_{1}}=\alpha_{R D_{2}}=2$, whereas Case 2 plots the variation of average BLERs versus $\alpha_{R D_{1}}=\alpha_{R D_{2}}$ with $\alpha_{S R}=2$. It can be inferred from Fig. $2(b)$ that the average BLER initially decreases and then saturates to a nearly fixed value with an increase in the value of shape factors. This is due to the fact that an increase in the value of the shape factor $\alpha_{Y}, Y \in\left\{S R, R D_{i}\right\}$, for fixed values of average channel gains $\lambda_{Y}=\frac{\alpha_{Y}}{\beta_{Y}}$, reduces the skew of the positively skewed distribution curve of the channel gain coefficient and results in an initial average BLER reduction at the users. However, a further increase in the value of $\alpha_{Y}$ leads to a significant decrease in the variance $\frac{\alpha_{Y}}{\beta_{Y}^{2}}$ of the corresponding fading coefficient. Consequently, the fading coefficient approaches the mean channel gain that is constant.

\section{A. Effect of Phase 1 and Phase 2 Blocklength on Average $B L E R$}

Fig. 3(a) and Fig. 3(b) show the BLER at users $D_{1}$ and $D_{2}$, respectively, versus the number of channel uses in the two communication phases. It can be inferred from the figure that the average BLERs at both the users $D_{1}, D_{2}$ decrease upon increasing the number of channel uses in the two phases of the communication process. However, the behavior with respect to the blocklength in each of the individual phases is quite different. In particular, the average BLERs at both the users decrease monotonically with respect to the number of channel uses in the first phase, denoted by $n_{1}$, while the number of channel uses in the second phase $n_{2}$ is held constant. This arises due to the fact that increasing $n_{1}$ results in a reduction of the average BLER together with an increase in the harvested energy at relay $R$, while the constant $n_{2}$ leads to higher transmission power for each transmission from the relay. As a result, the average BLER decreases monotonically at both the users. On the other hand, in the reverse scenario with $n_{1}$ being fixed and $n_{2}$ increasing, the rate of decrease is quite small in comparison to the previous case and appears to saturate at a particular BLER value. This is because for a 


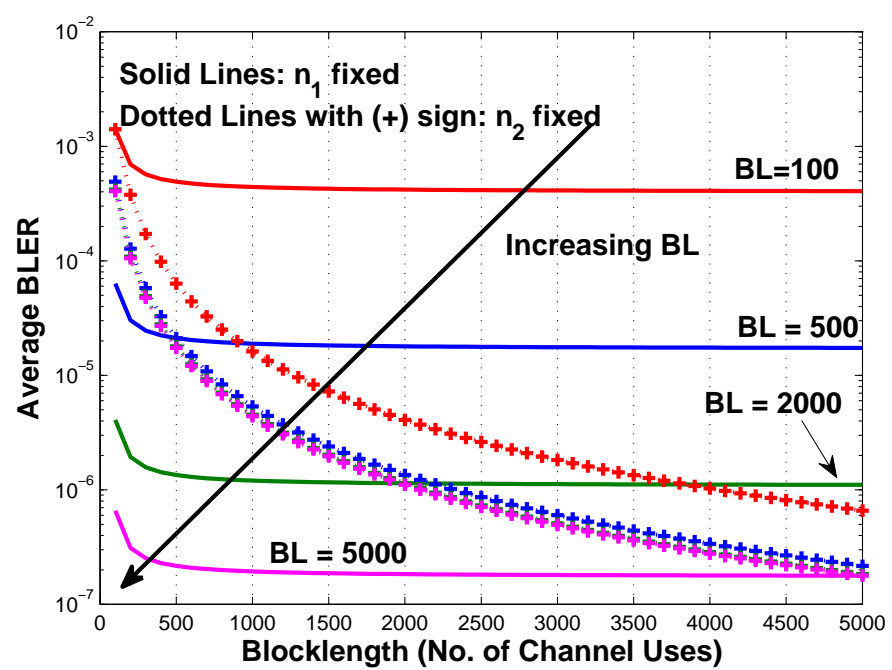

(a)

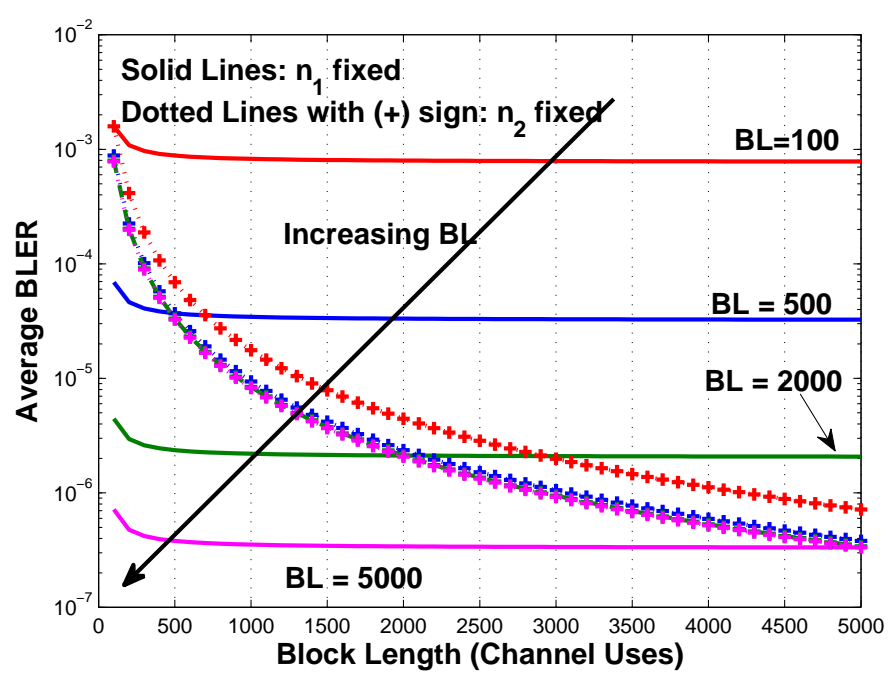

(b)

Fig. 3: (a) Average BLER versus blocklength (channel uses) performance at user $D_{1}$ (Eq. (18)). (b) Average BLER versus blocklength (channel uses) performance at user $D_{2}$ (Eq. (19)).

fixed $n_{1}$, the average BLER and the energy harvested at $R$ are constant. Thus, the reduction in average BLER in the second phase owing to increasing $n_{2}$ is offset by the increase in the average BLER due to the reduction in power available for each transmission from the relay to the two users.

\section{B. Effect of Splitting Factor $\mu$ on Average BLER}

Fig. 4(a) shows the average BLER performance at users $D_{1}, D_{2}$ versus the splitting factor $\mu$ at $\rho_{S}=20 \mathrm{~dB}$ for $N_{1}=N_{2}=50$ bits, $n_{1}=n_{2}=225, \lambda_{R D_{1}}=1, \lambda_{R D_{2}}=3$ and three different values of average source-relay channel gains $\lambda_{S R}=1,10,100$. It can be observed from the figures that the optimum value of the splitting factor for information decoding at the relay is inversely proportional to the quality of the source-relay channel. Since the relay is more likely to decode the information symbol correctly for a stronger sourcerelay channel, a higher energy assignment to the second phase of communication will lead to an improvement in the end-toend average BLER performance at the two users. However, in the scenario when the relay-destination channels are strong and the expected source-relay channel gain is constant at $\lambda_{S R}=5$ as shown in Fig. 4(b), a lower amount of energy would be required for transmission from the relay to destination. This necessitates an increase in the value of the splitting factor $\mu$ in order to reduce the end-to-end average BLER.

\section{Average BLER Performance and End-to-End Blocklength Minimization at High SNRs}

Fig. 5(a) presents the asymptotic average BLER performance for decoding $x_{1}$ at terminals $R, D_{1}, D_{2}$. The various parameters have been set as $a_{1}=b_{1}=0.8$ and $n_{1}=n_{2}=225$. Moreover, the splitting factor for information decoding has been set as $\mu=0.15$, since, it can be inferred from Fig. $4(a)$, that the optimum value of $\mu$ for the parameters chosen is in the range $\mu \leq 0.15$. A near-perfect overlap can be observed between the average BLER expressions for decoding $x_{1}$ at $U$, denoted by $\mathbb{E}\left(\tilde{\epsilon}_{1}^{U}\right)$, derived in Section III and the asymptotic ones denoted by $\mathbb{E}\left(\bar{\epsilon}_{1}^{U}\right)$, in Lemma 1, in Section IV, even at moderate SNR values. A similar overlap can be observed in Fig. 5(b) between the plots of the average BLER expressions for decoding $x_{2}$ at $R$ and at $D_{2}$ in the second phase of communication, at high SNR. In fact, while the analytical curve tightly approximates the average BLER for decoding $x_{1}$ at various terminals at all SNRs, there is only a slight deviation in Fig. 5(b) for decoding $x_{2}$ at very low SNRs. Recall that the corresponding analytical plots obtained using the asymptotic expressions of $\mathbb{E}\left(\bar{\epsilon}_{2}^{R}\right), \mathbb{E}\left(\bar{\epsilon}_{2}^{D_{2}}\right)$ have been derived in Lemma 2 , in Section IV. This confirms the accuracy of the results derived in Lemmas 1 and 2. Fig. 6(a) depicts the degradation in the average BLER performance for decoding symbol $x_{1}$ at terminal $U$, due to the interference from symbol $x_{2}$, at high SNR. A close match can be observed between the exact values and the closed-form asymptotic bound obtained in Theorem 2 .

Fig. $6(b)$ and $7(a)$ consider two different scenarios to plot the minimum blocklength obtained from the optimization framework in Section V of the paper for the SWIPT-based energy harvesting system and in Section IX of our technical report in [63] for the WET enabled energy harvesting system wherein wireless energy transfer and information transmission to the relay take place separately in the first and second phase, respectively, followed by the relay's broadcasting of the decoded superposed signal to the two users in the third phase. In particular, Scenario 1 considers reliability constraint at $D_{1}$ only that is $\epsilon_{1}^{t h}=10^{-5}, \epsilon_{2}^{t h}=1$ whereas in Scenario 2 , reliability constraints at both $D_{1}, D_{2}$ have been considered that is $\epsilon_{1}^{t h}=10^{-5}, \epsilon_{2}^{t h}=10^{-4}$. The parameters have been set as $N_{1}=N_{2}=256$. Moreover, for comparison, the performance of two OMA-based schemes are also shown in Fig. $6(b)$ and $7(a)$, where the optimization problem for the end-to-end blocklength minimization for the OMA-based SWIPT system is presented in Section VIII of our technical 


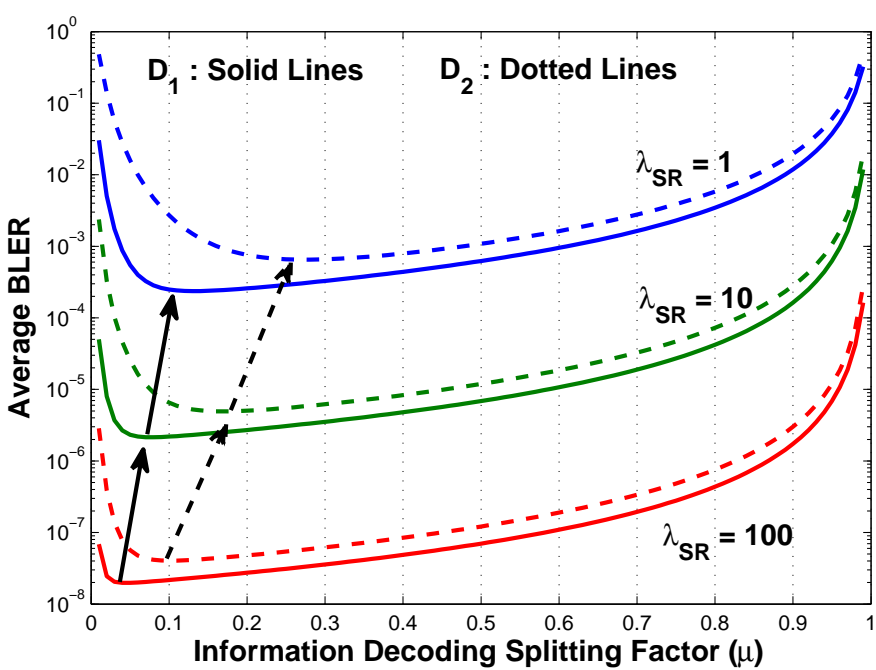

(a)

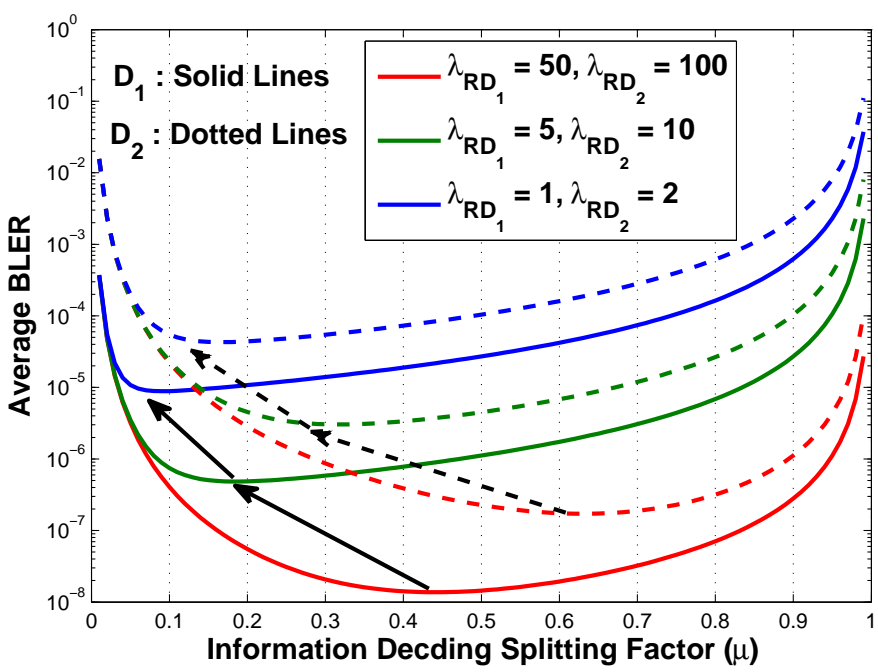

(b)

Fig. 4: (a) Average BLER versus splitting factor for information decoding $(\mu)$ performance with constant relay-destination average channel gains (Eq. (18),(19)). (b) Average BLER versus splitting factor for information decoding $(\mu)$ performance with constant source-relay average channel gain (Eq. (18),(19)).



(a)

Fig. 5: $(a)$ Asymptotic average BLER for decoding $x_{1}$ at $R, D_{1}, D_{2}$ for decoding $x_{2}$ at $R, D_{2}$ (Eq. (37)).

report in [63]. Scheme 1 considers two-user total power budget constraint stated as $a_{1}^{O M A}+a_{2}^{O M A} \leq 1$, whereas Scheme 2 considers the per transmission power constraints given by $a_{1}^{O M A} \leq 1, a_{2}^{O M A} \leq 1$, where $a_{1}^{O M A}$ and $a_{2}^{O M A}$ are the source power factors for symbol $x_{1}$ and $x_{2}$, respectively. It can be readily ascertained from the figures that the SWIPT based non-orthogonal scheme outperforms the three other techniques, requiring a substantially lower minimum blocklength for achieving the target integrity. Intuitively, this arises due to the fact that unlike the other competing schemes relying on three or four phases in total to transmit the message to both the users, the SWIPT based non-orthogonal scheme requires only two phases for completing the end-to-end communication from source to destination. Furthermore, a nearly overlapping

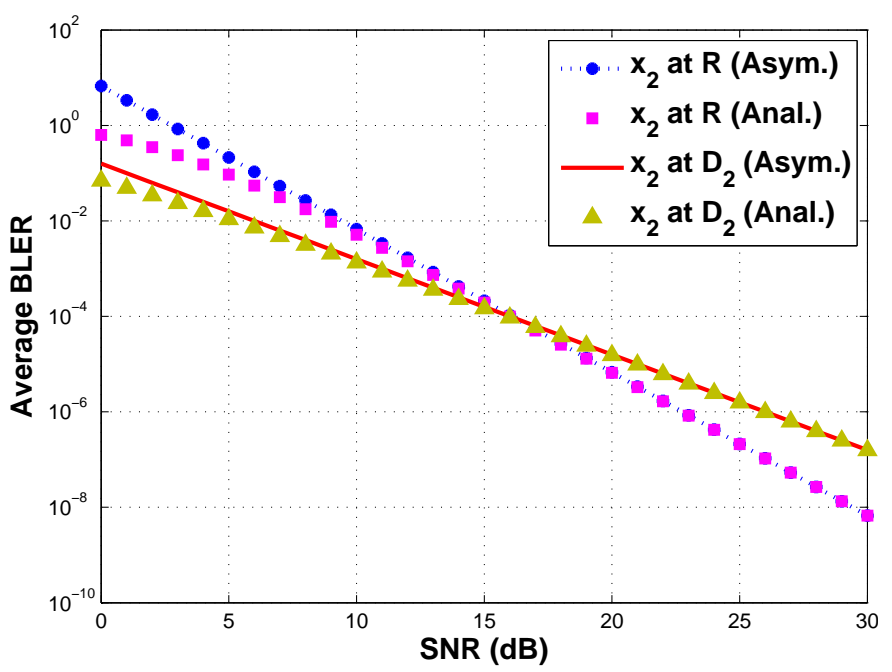

(b)

(Eq. (33)). (b) Asymptotic average BLER versus SNR performance

performance can be observed of the two OMA-based schemes from Fig. 6(b). This is because approximately no power will be allocated to $D_{2}$ in both OMA-based Scheme 1 and Scheme 2 when $\epsilon_{2}^{t h}=1$. Finally, Fig. $7(b)$. presents the minimum sum blocklength required versus the number of transmitted bits for the proposed as well as competing schemes, for two different values of SNR set as $\rho_{S}=20 \mathrm{~dB}$ and $\rho_{S}=25$ $\mathrm{dB}$. The figure once again bears testimony to the improved performance of the proposed scheme in comparison to other alternative communication protocols.

\section{CONCLUSIONS}

We have analyzed the performance of a non-orthogonal SWIPT enabled energy harvesting cooperative communication 


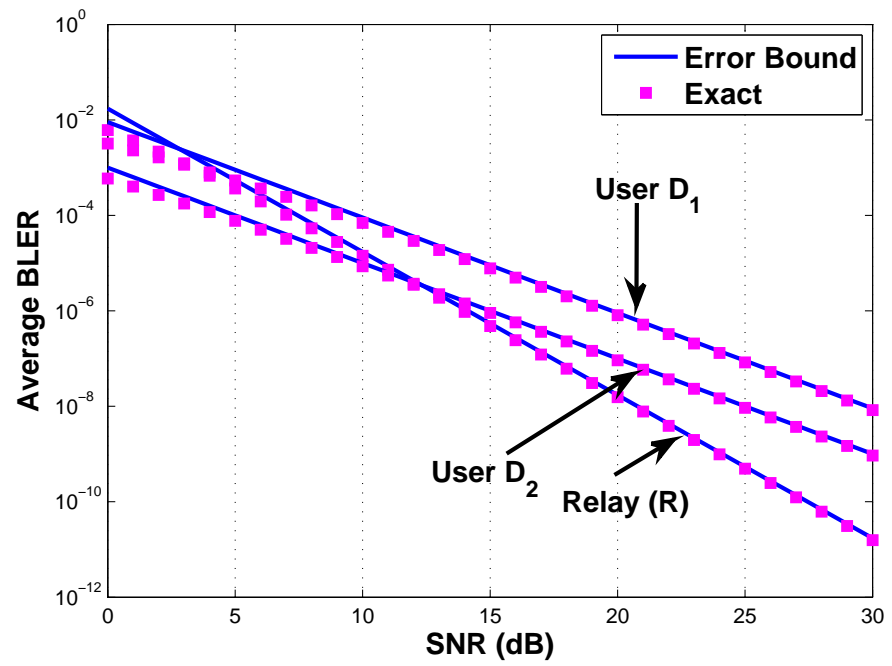

(a)

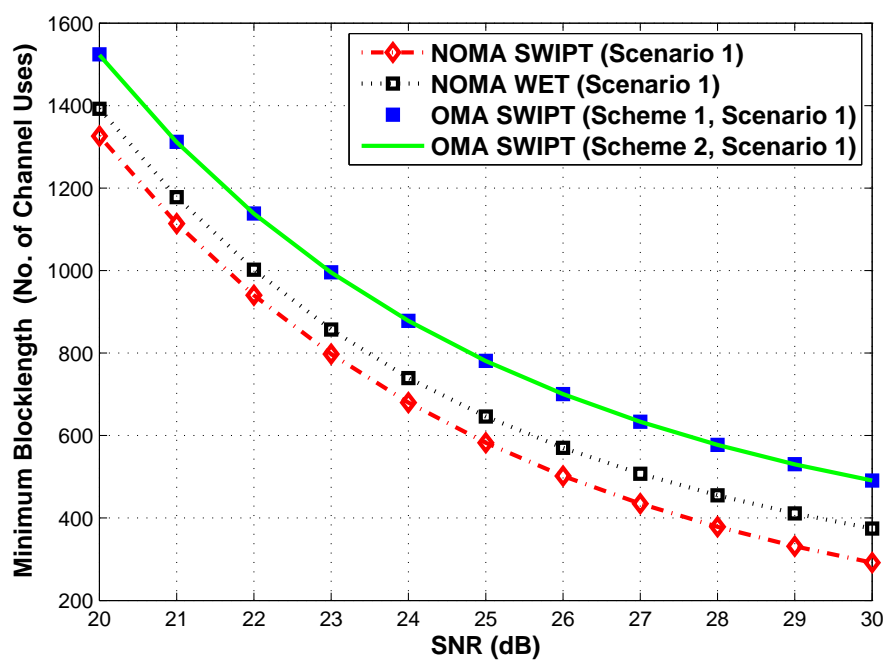

(b)

Fig. 6: $(a)$. Asymptotic average BLER due to interference from $x_{2}$ while decoding $x_{1}$ at $R, D_{1}, D_{2}$ versus SNR performance (Eq. (40)). (b) . Minimum sum-blocklength performance of the proposed joint splitting factor, user power allocation and blocklength minimization framework for our non-orthogonal SWIPT system (Eq. (52)), non-orthogonal WET ( [63], eq. (48)) and OMA-based Scheme 1 ( [63], eq. (40)), Scheme 2 ( [63], eq. (41)) versus SNR Scenario 1.

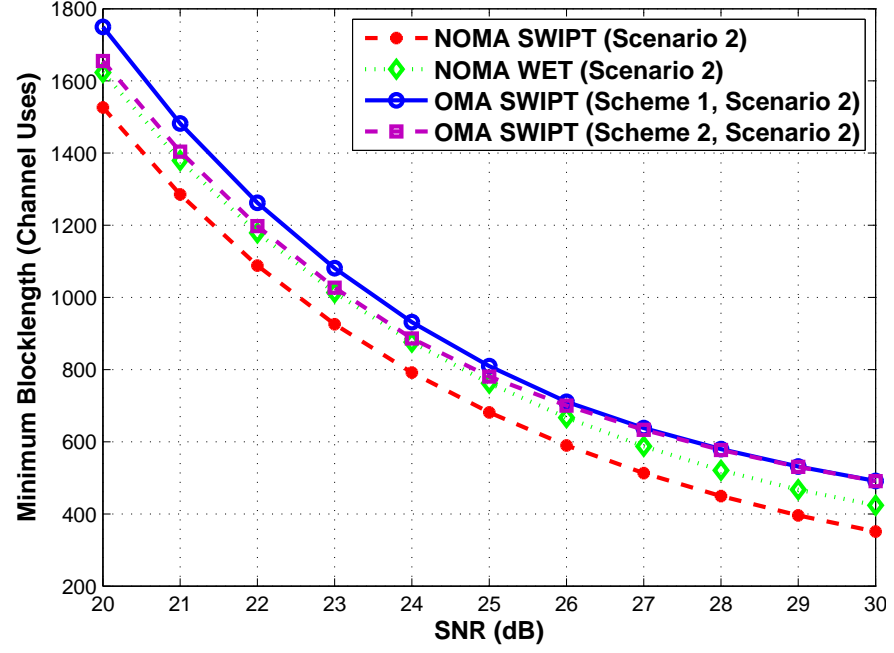

(a)

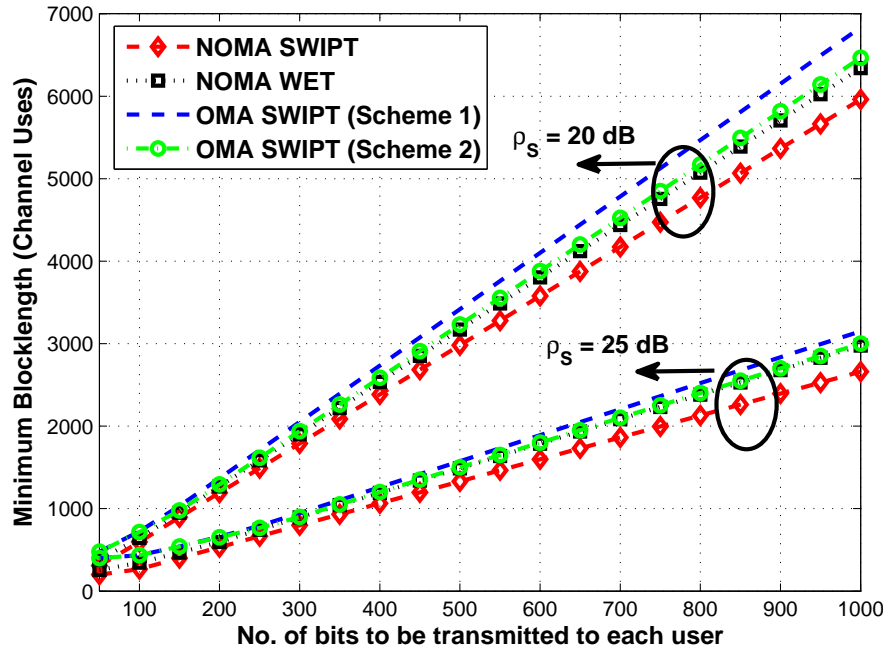

(b)

Fig. 7: Minimum sum-blocklength performance of the proposed joint splitting factor, user power allocation and blocklength minimization framework for our non-orthogonal SWIPT system (Eq. (52)), non-orthogonal WET ( [63], eq. (48)) and OMA-based Scheme 1 ( [63], eq. (40)), Scheme 2 ( [63], eq. (41)) versus (a). SNR and Scenario 2. (b). the number of user data bits.

system considering FBLs. Novel analytical expressions have been derived for the end-to-end average BLER considering a two-user relay-aided downlink communication scenario with no direct path between the source and the users. Novel expressions have also been obtained both for the asymptotic average end-to-end BLER and for the diversity order at the two users for potentially dissimilar fading links, followed by the explicit characterization of the effect of the interference due to the superposition of user signals on the average BLER performance of both the users. Finally, a novel joint optimization framework has also been developed for optimizing the user's power allocation and energy-splitting coefficient for end-toend blocklength minimization by transforming the original non-convex optimization problem into a GP. The proposed scheme has yielded a significantly improved performance in comparison to the non-orthogonal-WET and OMA powersplitting aided SWIPT-based schemes.

\section{REFERENCES}

[1] G. Durisi, T. Koch, and P. Popovski, "Toward massive, ultrareliable, and low-latency wireless communication with short packets," Proceedings of the IEEE, vol. 104, no. 9, pp. 1711-1726, 2016.

[2] C. Bockelmann, N. Pratas, H. Nikopour, K. Au, T. Svensson, C. Stefanovic, P. Popovski, and A. Dekorsy, "Massive machine-type communications in 5G: Physical and MAC-layer solutions," IEEE Communications Magazine, vol. 54, no. 9, pp. 59-65, 2016. 
[3] H. Chen, R. Abbas, P. Cheng, M. Shirvanimoghaddam, W. Hardjawana, W. Bao, Y. Li, and B. Vucetic, "Ultra-reliable low latency cellular networks: Use cases, challenges and approaches," IEEE Communications Magazine, vol. 56, no. 12, pp. 119-125, 2018.

[4] W. Shin, M. Vaezi, B. Lee, D. J. Love, J. Lee, and H. V. Poor, "Nonorthogonal multiple access in multi-cell networks: Theory, performance, and practical challenges," IEEE Communications Magazine, vol. 55, no. 10, pp. 176-183, 2017.

[5] L. Dai, B. Wang, Z. Ding, Z. Wang, S. Chen, and L. Hanzo, "A survey of non-orthogonal multiple access for 5G," IEEE Communications Surveys \& Tutorials, vol. 20, no. 3, pp. 2294-2323, 2018.

[6] L. Dai, B. Wang, Y. Yuan, S. Han, I. Chih-Lin, and Z. Wang, "Nonorthogonal multiple access for 5G: solutions, challenges, opportunities, and future research trends," IEEE Communications Magazine, vol. 53, no. 9, pp. 74-81, 2015.

[7] Y. Liu, Z. Qin, M. Elkashlan, Z. Ding, A. Nallanathan, and L. Hanzo, "Non-orthogonal multiple access for 5G and beyond," Proceedings of the IEEE, vol. 105, no. 12, pp. 2347-2381, 2017.

[8] Z. Ding, Z. Yang, P. Fan, and H. V. Poor, "On the performance of non-orthogonal multiple access in $5 \mathrm{G}$ systems with randomly deployed users," IEEE Signal Processing Letters, vol. 21, no. 12, pp. 1501-1505, 2014.

[9] J.-B. Kim and I.-H. Lee, "Capacity analysis of cooperative relaying systems using non-orthogonal multiple access," IEEE Communications Letters, vol. 19, no. 11, pp. 1949-1952, 2015.

[10] S. R. Islam, N. Avazov, O. A. Dobre, and K.-S. Kwak, "Power-domain non-orthogonal multiple access (NOMA) in 5G systems: Potentials and challenges," IEEE Communications Surveys \& Tutorials, vol. 19, no. 2, pp. 721-742, 2016.

[11] Y. Liu, H. Xing, C. Pan, A. Nallanathan, M. Elkashlan, and L. Hanzo, "Multiple-antenna-assisted non-orthogonal multiple access," IEEE Wireless Communications, vol. 25, no. 2, pp. 17-23, 2018.

[12] Z. Ding, F. Adachi, and H. V. Poor, "The application of MIMO to non-orthogonal multiple access," IEEE Transactions on Wireless Communications, vol. 15, no. 1, pp. 537-552, 2016.

[13] Z. Ding, R. Schober, and H. V. Poor, "A general MIMO framework for NOMA downlink and uplink transmission based on signal alignment," IEEE Transactions on Wireless Communications, vol. 15, no. 6, pp. 4438-4454, 2016.

[14] Z. Q. Al-Abbasi and D. K. So, "Resource allocation in non-orthogonal and hybrid multiple access system with proportional rate constraint," IEEE Transactions on Wireless Communications, vol. 16, no. 10, pp. 6309-6320, 2017.

[15] Z. Ding, M. Peng, and H. V. Poor, "Cooperative non-orthogonal multiple access in 5G systems," IEEE Communications Letters, vol. 19, no. 8, pp. 1462-1465, 2015.

[16] Y. Li, M. Jiang, Q. Zhang, Q. Li, and J. Qin, "Cooperative nonorthogonal multiple access in multiple-input-multiple-output channels,' IEEE Transactions on Wireless Communications, vol. 17, no. 3, pp. 2068-2079, 2018

[17] C. Xue, Q. Zhang, Q. Li, and J. Qin, "Joint power allocation and relay beamforming in nonorthogonal multiple access amplify-and-forward relay networks," IEEE Transactions on Vehicular Technology, vol. 66, no. 8, pp. 7558-7562, 2017.

[18] X. Yue, Y. Liu, S. Kang, A. Nallanathan, and Y. Chen, "Modeling and analysis of two-way relay non-orthogonal multiple access systems," IEEE Transactions on Communications, vol. 66, no. 9, pp. 3784-3796, 2018.

[19] A. Agarwal and A. K. Jagannatham, "Performance analysis for nonorthogonal multiple access (NOMA)-based two-way relay communication," IET Communications, vol. 13, no. 4, pp. 363-370, 2019.

[20] C. E. Shannon, "A mathematical theory of communication," $A C M$ SIGMOBILE mobile computing and communications review, vol. 5, no. 1, pp. 3-55, 2001.

[21] X. Zhang, Q. Zhu, and H. V. Poor, "Non-asymptotic performance for finite blocklength coding over Nakagami- $m$ channels," in Proc. IEEE International Conference on Communications (ICC), 2019, pp. 1-6.

[22] Y. Polyanskiy, H. V. Poor, and S. Verdú, "Channel coding rate in the finite blocklength regime," IEEE Transactions on Information Theory, vol. 56, no. 5, pp. 2307-2359, 2010.

[23] Y. Xu, C. Shen, T.-H. Chang, S.-C. Lin, Y. Zhao, and G. Zhu, "Energyefficient non-orthogonal transmission under reliability and finite blocklength constraints," in Proc. IEEE Globecom Workshops (GC Wkshps), 2017, pp. 1-6.

[24] Y. Yu, H. Chen, Y. Li, Z. Ding, and B. Vucetic, "On the performance of non-orthogonal multiple access in short-packet communications," IEEE Communications Letters, vol. 22, no. 3, pp. 590-593, 2018.
[25] X. Sun, S. Yan, N. Yang, Z. Ding, C. Shen, and Z. Zhong, "Shortpacket downlink transmission with non-orthogonal multiple access," IEEE Transactions on Wireless Communications, vol. 17, no. 7, pp. 4550-4564, 2018

[26] M. Amjad and L. Musavian, "Performance analysis of NOMA for ultrareliable and low-latency communications," in Proc. IEEE Globecom Workshops (GC Wkshps). IEEE, 2018, pp. 1-5.

[27] X. Huang and N. Yang, "On the block error performance of short-packet non-orthogonal multiple access systems," in Proc. IEEE International Conference on Communications (ICC). IEEE, 2019, pp. 1-7.

[28] J. Zheng, Q. Zhang, and J. Qin, "Average block error rate of downlink NOMA short-packet communication systems in Nakagami- $m$ fading channels," IEEE Communications Letters, vol. 23, no. 10, pp. 1712 1716, 2019.

[29] Y. Hu, J. Gross, and A. Schmeink, "On the performance advantage of relaying under the finite blocklength regime," IEEE Communications Letters, vol. 19, no. 5, pp. 779-782, 2015.

[30] _ - "On the capacity of relaying with finite blocklength," IEEE Transactions on Vehicular Technology, vol. 65, no. 3, pp. 1790-1794, 2016.

[31] Y. Li, M. C. Gursoy, and S. Velipasalar, "Throughput of two-hop wireless channels with queueing constraints and finite blocklength codes," in Proc. IEEE International Symposium on Information Theory (ISIT). IEEE, 2016, pp. 2599-2603.

[32] Y. Hu, A. Schmeink, and J. Gross, "Blocklength-limited performance of relaying under quasi-static Rayleigh channels," IEEE Transactions on Wireless Communications, vol. 15, no. 7, pp. 4548-4558, 2016.

[33] Y. Hu, C. Schnelling, M. C. Gursoy, and A. Schmeink, "Multi-relayassisted low-latency high-reliability communications with best single relay selection," IEEE Transactions on Vehicular Technology, vol. 68, no. 8, pp. 7630-7642, 2019.

[34] X. Lai, Q. Zhang, and J. Qin, "Cooperative NOMA short-packet communications in flat Rayleigh fading channels," IEEE Transactions on Vehicular Technology, vol. 68, no. 6, pp. 6182-6186, 2019.

[35] A. Zanella, N. Bui, A. Castellani, L. Vangelista, and M. Zorzi, "Internet of things for smart cities," IEEE Internet of Things Journal, vol. 1, no. 1, pp. 22-32, 2014.

[36] J. Hu, K. Yang, G. Wen, and L. Hanzo, "Integrated data and energy communication network: A comprehensive survey," IEEE Communications Surveys \& Tutorials, vol. 20, no. 4, pp. 3169-3219, 2018.

[37] X. Lu, P. Wang, D. Niyato, D. I. Kim, and Z. Han, "Wireless charging technologies: Fundamentals, standards, and network applications," IEEE Communications Surveys \& Tutorials, vol. 18, no. 2, pp. 1413-1452, 2016.

[38] I. Krikidis, S. Timotheou, S. Nikolaou, G. Zheng, D. W. K. Ng, and R. Schober, "Simultaneous wireless information and power transfer in modern communication systems," IEEE Communications Magazine, vol. 52, no. 11, pp. 104-110, 2014.

[39] A. A. Nasir, X. Zhou, S. Durrani, and R. A. Kennedy, "Relaying protocols for wireless energy harvesting and information processing," IEEE Transactions on Wireless Communications, vol. 12, no. 7, pp. 3622-3636, 2013.

[40] X. Lu, P. Wang, D. Niyato, D. I. Kim, and Z. Han, "Wireless networks with RF energy harvesting: A contemporary survey," IEEE Communications Surveys \& Tutorials, vol. 17, no. 2, pp. 757-789, 2015.

[41] L. R. Varshney, "Transporting information and energy simultaneously," in Proc. IEEE International Symposium on Information Theory (ISIT), 2008, pp. 1612-1616.

[42] R. Zhang, R. G. Maunder, and L. Hanzo, "Wireless information and power transfer: from scientific hypothesis to engineering practice," IEEE Communications Magazine, vol. 53, no. 8, pp. 99-105, 2015.

[43] X. Zhou, R. Zhang, and C. K. Ho, "Wireless information and power transfer: Architecture design and rate-energy tradeoff," IEEE Transactions on Communications, vol. 61, no. 11, pp. 4754-4767, 2013.

[44] H. Chen, Y. Li, J. L. Rebelatto, B. F. Uchôa Filho, and B. Vucetic, "Harvest-then-cooperate: Wireless-powered cooperative communications." IEEE Transactions on Signal Processing, vol. 63, no. 7, pp. 1700-1711, 2015.

[45] X. Zhou, J. Li, F. Shu, Q. Wu, Y. Wu, W. Chen, and L. Hanzo, "Secure SWIPT for directional modulation-aided AF relaying networks," IEEE Journal on Selected Areas in Communications, vol. 37, no. 2, pp. 253268, 2019.

[46] O. L. A. López, E. M. G. Fernández, R. D. Souza, and H. Alves, "Wireless powered communications with finite battery and finite blocklength," IEEE Transactions on Communications, vol. 66, no. 4, pp. 1803-1816, 2017. 
[47] O. L. A. López, H. Alves, R. D. Souza, and E. M. G. Fernández, "Ultrareliable short-packet communications with wireless energy transfer," IEEE Signal Processing Letters, vol. 24, no. 4, pp. 387-391, 2017.

[48] O. L. A. López, E. M. G. Fernández, R. D. Souza, and H. Alves, "Ultrareliable cooperative short-packet communications with wireless energy transfer," IEEE Sensors Journal, vol. 18, no. 5, pp. 2161-2177, 2018.

[49] B. Makki, T. Svensson, and M. Zorzi, "Wireless energy and information transmission using feedback: Infinite and finite block-length analysis," IEEE Transactions on Communications, vol. 64, no. 12, pp. 5304-5318, 2016.

[50] T. A. Khan, R. W. Heath, and P. Popovski, "Wirelessly powered communication networks with short packets," IEEE Transactions on Communications, vol. 65, no. 12, pp. 5529-5543, 2017.

[51] O. L. A. López, R. D. Souza, H. Alves, and E. M. G. Fernández, "Ultra reliable short message relaying with wireless power transfer," in Proc. IEEE International Conference on Communications (ICC), 2017, pp $1-6$.

[52] M. Haghifam, B. Makki, M. Nasiri-Kenari, T. Svensson, and M. Zorzi, "Wireless-powered relaying with finite block-length codes," arXiv preprint arXiv:1611.05995, 2016.

[53] Y. Hu, Y. Zhu, M. C. Gursoy, and A. Schmeink, "SWIPT-enabled relaying in IoT networks operating with finite blocklength codes," IEEE Journal on Selected Areas in Communications, vol. 37, no. 1, pp. 74-88, 2018.

[54] J. Tang, J. Luo, M. Liu, D. K. So, E. Alsusa, G. Chen, K.-K. Wong, and J. A. Chambers, "Energy efficiency optimization for NOMA with SWIPT," IEEE Journal of Selected Topics in Signal Processing, vol. 13, no. 3, pp. 452-466, 2019 .

[55] M. Yuksel and E. Erkip, "Broadcast strategies for the fading relay channel," in Proc. IEEE Military Communications Conference (MILCOM), 2004, pp. 1060-1065.

[56] C. Luo, G. Parr, S. I. McClean, C. Peoples, and X. Wang, "Hybrid demodulate-forward relay protocol for two-way relay channels," IEEE Transactions on Wireless Communications, vol. 14, no. 8, pp. 4328 4341, 2015

[57] M. M. Amiri and B. Maham, "Two novel adaptive transmission schemes in a decode-and-forward relaying network," Wireless Personal Communications, vol. 96, no. 4, pp. 5705-5722, 2017.

[58] D. Wan, M. Wen, F. Ji, H. Yu, and F. Chen, "Non-orthogonal multiple access for cooperative communications: Challenges, opportunities, and trends," IEEE Wireless Communications, vol. 25, no. 2, pp. 109-117, 2018.

[59] D. Wan, M. Wen, F. Ji, Y. Liu, and Y. Huang, "Cooperative NOMA systems with partial channel state information over Nakagami- $m$ fading channels," IEEE Transactions on Communications, vol. 66, no. 3, pp. 947-958, 2018.

[60] T. Hou, X. Sun, and Z. Song, "Outage performance for non-orthogonal multiple access with fixed power allocation over Nakagami- $m$ fading channels," IEEE Communications Letters, vol. 22, no. 4, pp. 744-747, 2018.

[61] C. Peng, F. Li, and H. Liu, "Optimal power splitting in two-way decodeand-forward relay networks," IEEE Communications Letters, vol. 21, no. 9, pp. 2009-2012, 2017.

[62] Y. Dong, M. J. Hossain, and J. Cheng, "Performance of wireless powered amplify and forward relaying over Nakagami- $m$ fading channels with nonlinear energy harvester," IEEE Communications Letters, vol. 20, no. 4, pp. 672-675, 2016.

[63] A. Agarwal, A. K. Jagannatham, and L. Hanzo, "Technical Report: Finite blocklength non-orthogonal cooperative communication relying on SWIPT-enabled energy harvesting relays." IIT Kanpur, Kanpur, India, Technical Report, 2019. [Online]. Available: http:www.iitk.ac.in/ mwn/documents/TR_NOMA_FBL_SWIPT_19_v2.pdf.

[64] B. Makki, T. Svensson, and M. Zorzi, "Finite block-length analysis of the incremental redundancy HARQ," IEEE Wireless Communications Letters, vol. 3, no. 5, pp. 529-532, 2014.

[65] L. Comtet, Advanced Combinatorics: The art of finite and infinite expansions. Springer Science \& Business Media, 2012.

[66] I. S. Gradshteyn and I. M. Ryzhik, Table of integrals, series, and products. Academic press, 2014.

[67] J. Zhu, J. Wang, Y. Huang, S. He, X. You, and L. Yang, "On optimal power allocation for downlink non-orthogonal multiple access systems," IEEE Journal on Selected Areas in Communications, vol. 35, no. 12, pp. 2744-2757, 2017.

[68] P. Mary, J.-M. Gorce, A. Unsal, and H. V. Poor, "Finite blocklength information theory: What is the practical impact on wireless communications?" in Proc. IEEE Globecom Workshops (GC Wkshps), 2016, pp. $1-6$.
[69] S. Boyd and L. Vandenberghe, Convex optimization. Cambridge university press, 2004.

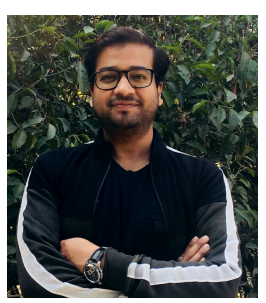

Akash Agarwal (S'16) received his B.Tech. and M.E. degrees in Electronics and Communication from Jaypee University of I.T., Solan and Thapar University, Patiala, India, respectively. $\mathrm{He}$ is currently working toward the Ph.D. degree in the Dept. of Electrical Engineering at Indian Institute of Technology Kanpur, Kanpur, India. His research interests include NOMA, Energy Harvesting, Cooperative communication and Signal Processing for 5G multimedia networks.

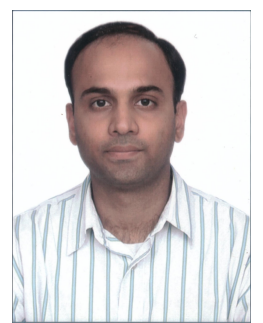

Aditya K. Jagannatham received his Bachelors degree from the Indian Institute of Technology, Bombay and M.S. and Ph.D. degrees from the University of California, San Diego, U.S.A. From April '07 to May '09 he was employed as a senior wireless systems engineer at Qualcomm Inc., San Diego, California, where he was a part of the Qualcomm CDMA technologies (QCT) division. His research interests are in the area of next-generation wireless cellular and WiFi networks, with special emphasis on various $5 \mathrm{G}$ technologies such as massive MIMO, mmWave MIMO, FBMC, NOMA, Full Duplex and others. He is currently a Professor in the Electrical Engineering department at IIT Kanpur, where he holds the Arun Kumar Chair Professorship, and is also associated with the BSNL-IITK Telecom Center of Excellence (BITCOE). He has been twice awarded the P.K. Kelkar Young Faculty Research Fellowship for excellence in research, the Qualcomm Innovation Fellowship (QInF) and the IIT Kanpur Excellence in Teaching Award. He was awarded the CAL(IT)2 fellowship at the University of California San Diego and the Upendra Patel Achievement Award at Qualcomm.

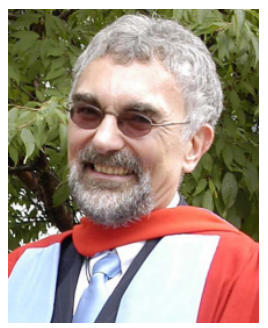

Lajos Hanzo (M'91-SM'92-F'04) (https://en.wikip edia.org/wiki/Lajos_Hanzo) received his 5-year Master degree in Electronics in 1976 and his doctoral degree in 1983. In 2004 the University of Southampton bestowed the Doctor of Science (DSc) higher research degree upon him. During his 44year career in Telecommunications, he has held various research and academic positions in Hungary, Germany, and U.K. Since 1986, he has been with the School of Electronics and Computer Science, University of Southampton, U.K., where he holds the Chair of Telecommunications. He has successfully supervised 119 Ph.D. students, coauthored $18 \mathrm{John}$ Wiley/IEEE Press books on mobile radio communications, totaling in excess of 10000 pages, published 1329 research papers at IEEE Xplore, acted as TPC and the General Chair of IEEE conferences, presented keynote lectures, and has been awarded a number of distinctions. He is currently directing an academic research team, working on a range of research projects in the field of wireless multimedia communications, sponsored by industry, the Engineering and Physical Sciences Research Council (EPSRC), U.K., the European Research Council's Advanced Fellow Grant, and the Royal Society, UK. He is an enthusiastic supporter of industrial and academic liaison, and he offers a range of industrial courses. He is a Fellow of the Royal Academy of Engineering (FREng), Fellow of the Institute of Engineering and Technology (FIET), Fellow of the European Signal Processing Association (EURASIP). In 2009, he received an Honorary Doctorate from the Technical University of Budapest, and again in 2015 by from University of Edinburgh. From 2008 to 2012, he was the Editor-in-Chief of the IEEE Press and a Chaired Professor at Tsinghua University, Beijing. His research is funded by the European Research Council's Advanced Fellow Grant. He is a Governor of the IEEE VTS. 